City University of New York (CUNY)

CUNY Academic Works

\title{
A COMPARISON OF HALF AND QUARTER SPACE PENETRATION INTO GRANULAR MEDIA
}

Ivan L. Guzman

CUNY New York City College of Technology

Magued Iskander

New York University

Stephan Bless

New York University

\section{How does access to this work benefit you? Let us know!}

More information about this work at: https://academicworks.cuny.edu/ny_pubs/767

Discover additional works at: https://academicworks.cuny.edu

This work is made publicly available by the City University of New York (CUNY).

Contact: AcademicWorks@cuny.edu 


\title{
A COMParison OF HALF AND QUARTER SPACE PENETRATION INTO GranUlar MEDia
}

\author{
Ivan L. Guzman, $\mathrm{PhD}, \mathrm{PE}^{1}$, Magued Iskander, $\mathrm{PhD}, \mathrm{PE}^{2}$, Stephan Bless, $\mathrm{ScD}^{3}$
}

\begin{abstract}
In this study, two experimental techniques are compared, for the purpose of visualizing projectile penetration at speeds ranging between 60 and $150 \mathrm{~m} / \mathrm{s}$ into granular media. The two techniques are half space penetration into a transparent synthetic soil surrogate and quarter space penetration of an opaque natural sand and transparent soil surrogate, against an observation window. In both techniques a pneumatic projectile accelerator was employed to launch the projectiles and high-speed imagery was employed to visualize the penetration events, un-intrusively. Transparency in transparent targets was achieved by saturating angular fused quartz with a refractive index matched pore fluid made of sucrose. Comparison of both techniques suggests that their results are complimentary. In particular, the terminal depth of penetration is not significantly inhibited by shooting in quarter space. Additionally, both techniques permitted visualizing distinct dilations zones ahead and around penetration. Each technique offers a number of distinct advantages. In particular, half space penetration reduces the possibility of projectile diversion and is much safer, while quarter space penetration allows for visualizing penetration into opaque targets at a finer scale than that achieved in half space penetration.
\end{abstract}

Keywords: Transparent soil, granular, penetration, half space, quarter space, dilation

${ }^{1}$ Assistant Professor, Construction Management and Civil Engineering Technology, New York City College of Technology, Brooklyn, NY, USA, iguzman@citytech.cuny.edu

2 Professor \& Chair, Civil and Urban Engineering Department, New York University, Tandon School of Engineering. Brooklyn, NY, USA, iskander@nyu.edu, (Corresponding Author)

${ }^{3}$ Research Professor, Civil and Urban Engineering Department, New York University, Tandon School of Engineering, Brooklyn NY, USA, sbless@nyu.edu 


\section{INTRODUCTION}

The dynamics of a penetrator traveling through a soil target is a subject of great interest that has been covered in several recent review articles (Omidvar, Iskander, and Bless 2012, 2014, Iskander, Bless, and Omidvar 2015). Literature on rapid penetration into granular media spans a number of disciplines including geotechnical engineering, soft matter physics, engineering geology, ballistics, petroleum engineering, and aerospace engineering. Over the past half century, treatment of the problem has evolved from one of empirical nature to semiempirical models based on experimentally verified phenomenology. Most experiments involved post-mortem evaluation of penetration events, based mainly on depth of burial. In the last decade, with advances in numerical modelling, attention has shifted from macroscale observations to exploration of underlying meso-scale and, to some degree, micro-scale interactions necessary to calibrate numerical models. Two visualization techniques have been developed to gain insights into meso-scale phenomena occurring during penetration. These are referred to collectively as (1) Quarter Space and (2) Half Space methods.

Quarter Space Methods: The penetration process through opaque materials may be visualized by use of a transparent observation window (rigid wall) adjacent to the trajectory. Evolution of explosive craters in sand was extensively studied with this technique by (Piekutowski 1980). The method was also applied to penetration of sand by (Borg and Vogler 2008, Borg et al. 2013) wherein a spherical projectile penetrates a sand target along the side of a rigid observation window. Using the terminology of Piekutowski, the conventional "half space" has been replaced by a "quarter space", (fig.1). Ideally to maintain symmetry the projectile should be sectioned along its length, resulting in a semicircular cross section, and shot so that the flat side runs against the observation window. However, this greatly complicates the experiment, and neither we, nor Borg and Vogler took this approach. Since we have true half space data (Guzman, Iskander, and Bless 2015) from similar impacts, we are able to qualitatively and quantitatively evaluate the extent to which presence of an observation window affects the projectile behavior and trajectory.

Half Space Methods: Naturally occurring soils are opaque, so researchers have developed synthetic transparent soil surrogates to visualize in situ meso-scale interactions between 
soil particles subjected to various types of loading, un-intrusively (Iskander 2010, Iskander 2016). These materials have been used to study penetration into synthetic soils (Chen et al. 2014, Omidvar et al. 2015). The particular family of transparent soils used in this study are made by carefully matching the refractive index (RI) of a granular synthetic transparent soil surrogate, fused quartz (RI = 1.457), with the RI of the saturating pore fluid, sucrose (Guzman et al. 2013, Guzman and Iskander 2013), (fig.2). The granular fused quartz is from the same manufacturer and has similar gradation to the fused quartz labeled, FQ-1 in (Guzman et al. 2013, Guzman and Iskander 2013). These studies as well as others (Ezzein and Bathurst 2011, Ferreira and Zornberg 2015) have shown that granular fused quartz has similar quasi-static rheological and geotechnical properties (shear strength, compressibility, particle breakage and grain size distribution) to natural sands.

Although both quarter and half space methods provide important insights into penetration mechanics, neither is ideal. Quarter space methods alter the problem's boundary conditions, while half space methods employ artificial, rather than natural soils. The objective of this study is to compare the results inferred from both approaches, identify where each method excels, and the degree to which they agree. The scope of this investigation involved capturing projectile penetration events into sucrose saturated, water saturated, and air saturated (dry) fused quartz (FQ), and air saturated (dry) and water saturated Ottawa sand. Sucrose saturated FQ is transparent and thus results in transparent targets suitable for both quarter space and half space methods while the other materials result in opaque targets, suitable for quarter space investigations only.

\section{EXPERIMENTAL SETUP}

\section{$2.1 \quad$ Materials}

Commercially sourced Ottawa Sand having an average particle size $D_{50}$ of $0.35 \mathrm{~mm}$ was used in this study. Maximum and minimum dry densities of Ottawa sand were 1.90 and 1.58 $\mathrm{g} / \mathrm{cm}^{3}$, respectively. Granular FQ having $D_{50}$ of $0.43 \mathrm{~mm}$ was used in this study. Maximum and minimum dry densities of FQ were determined experimentally with standardized laboratory procedures (ASTM D4253-16, Standard Test Methods for Maximum Index 
Density and Unit Weight of Soils Using a Vibratory Table, and ASTM D4254-16, Standard Test Methods for Minimum Index Density and Unit Weight of Soils and Calculation of Relative Density, respectively) to be 1.30 and $1.00 \mathrm{~g} / \mathrm{cm}^{3}$, respectively. The relative density scale is generally used to characterize the state of compaction of granular materials. All of the targets where tested at a relative density between 58 to $68 \%$.

FQ grains can generally be described as angular with high to low sphericity, according to Powers roundness and sphericity scale (Powers 1953), while Ottawa sand grains can be described as rounded with high sphericity. Drained triaxial tests indicate that peak angles of internal friction for dry and sucrose saturated FQ at different relative densities range between $47^{\circ}$ and $57^{\circ}$ (Guzman et al. 2013). These values are typical for angular sands (Holtz, Kovaks, and T.C. 2010). Peak angles of internal friction for Ottawa sand are reported to be $28^{\circ}$ and $35^{\circ}$ at loose and dense relative densities, respectively (Casagrande 1936). Dynamic viscosities for sucrose and water are reported to be 0.2012 and $0.0009 \mathrm{~Pa}$ s, respectively.

\section{$2.2 \quad$ Targets}

Quarter space specimens were prepared in $16.5 \mathrm{~cm}$ x $16.5 \mathrm{~cm}$ x $23.1 \mathrm{~cm}(6.5$ " x 6.5" x 9.1") (length $\mathrm{x}$ width $\mathrm{x}$ depth) acrylic containers. Due to the close proximity of the penetrating projectile to the observation window, we anticipated that the observation window could be gouged or damaged during the penetration event. Thus, as a precautionary measure the containers were fitted with a removable wall that could easily be replaced in the event that the projectile damaged or scratched the chamber wall. Foreseeing that having a removable lateral wall in a container could cause sealing problems when preparing saturated targets, the container was fitted with an inner removable wall. Two channels where permanently attached close to the container walls where a removable acrylic section (removable wall) could be inserted, (fig. 3). Quarter space penetration events were then conducted along the removable wall, which provides a photographic window into the event. Half space penetration shots were conducted in the middle of acrylic containers of similar dimensions.

Target preparation was conducted using similar techniques presented in (Guzman et al. 2014, Guzman, Iskander, and Bless 2015). Targets were prepared by pluviation (raining) of 
the soil particles into acrylic transparent containers. Pluviation of dry FQ and dry Ottawa sand targets commonly yielded a medium dense relative density, thus no extra compaction was needed. Preparation of water saturated FQ and Ottawa sand specimens was conducted by dry pluviation of the particles followed by slow flooding of the specimens with water until they were completely saturated. The saturating fluid was introduced at the bottom of the containers and allowed to rise slowly to displace air. If flooding is conducted slowly the particle arrangement is not altered, thus, pluviation followed by flooding yielded a medium dense relative density. Sucrose saturated transparent specimens were prepared by pluviation of the dry particles through several centimeters of sucrose saturating fluid. Pluviation through the saturating fluid creates a soil structure that mimics natural sedimentation. Also, the standing layer of liquid "filters" air bubbles, which is a major source of transparency degradation, thereby, improving the transparency of the specimens. This technique produced very loose specimens with a relative density of 10 to $15 \%$. To produce medium dense sucrose saturated transparent specimens, pluviation through sucrose was followed by vibrating the specimens until the desired relative density was achieved.

Vibration of the specimens was achieved using an electro-mechanical vibrator (Fritsch model Analysette 3 Pro), operated at an amplitude of $0.1-0.4 \mathrm{~mm}$. Vibration was conducted until a known quantity of soil occupied a predetermined volume previously marked on the transparent containers, thus ensuring the desired relative density. The weight of the container (plus saturating liquid) was recorded prior to and after pluviation to determine the weight fraction of solids deposited. The weight of the solid particles divided by the predetermined volume marked on the container (where the specimen was vibrated to) yielded the dry density of the saturated specimens; thus, the relative density of the saturated specimens could be calculated. The total (bulk) density for saturated specimens was then calculated by assuming $100 \%$ saturation. For dry specimens the total (bulk) density is the same as the dry density. A summary of the properties of the targets tested in this study is presented in table 1. 


\subsection{Projectile Accelerator and Projectiles}

The projectiles and projectile accelerator used in this study were the same as in (Guzman, Iskander, and Bless 2015). Type 316 stainless steel spherical projectiles were used in every test. The projectiles had a diameter of $9.9 \mathrm{~mm}(0.39 \mathrm{in})$, and a density of $7.6 \mathrm{~g} / \mathrm{cm}^{3}$, which yields a mass to diameter ratio of $3.9 \mathrm{~g} / \mathrm{cm}$.

Projectiles were propelled vertically downward using an electro-pneumatic projectile accelerator (Cave et al. 2014), at approximate entry velocities of 60, 100 and $150 \mathrm{~m} / \mathrm{s}$ (fig. 4). A custom-made optical speedometer attached to the barrel was used to measure projectile exit velocities. The speedometer consists of three infrared photo-sensor pairs fixed $30 \mathrm{~mm}$ apart mounted on a $150 \mathrm{~mm}$ long extension attached to the end of the barrel. As a projectile traveled past each sensor, a status change with an individual time stamp was recorded. The time between status changes in each sensor divided by the distance between the sensors yields the average speed of the projectile. This resulted in three reported speeds, between sensor 1 and 2, sensor 2 and 3, and sensor 1 and 3, which provided a measure of redundancy. Probable uncertainties of 1.1\%, 1.9\%, and 2.8\% were reported for speeds of 60 , 100, and $150 \mathrm{~m} / \mathrm{s}$, respectively (Cave et al. 2014). As an additional measure of redundancy, velocities were also calculated by measuring the distance traveled by the projectile, measured in pixels, before impact between consecutive camera frames, divided by the time between frames. The percent difference between the velocities measured by the speedometer and the ones measured by observing the projectile in consecutive frames varied between $-3.8 \%$ to $6.4 \%$. The differences also confirm that the drop-off in projectile velocity was not significant between the end of the barrel and the specimens.

The distance from the end of the barrel to the surface of the soil target was empirically determined to be greater than $23 \mathrm{~cm}$ in all shots. This allows for the air travelling ahead of, or behind of, the projectile (air blast) to disperse before reaching the soil target and thus, reduces its effect. Figure 5 shows an image of a projectile as it is about to penetrate a quarter space specimen at the highest speeds used in this study. As can be seen in the figure the effects of air blast were minimal. 
Test setup of quarter and half space tests presented different challenges. A significant degree of care was required to setup quarter space tests. In particular, both the accelerator and the observation window needed to be in perfect alignment, with the gravity axis. Half space tests in transparent targets required care to prevent loss of transparency by ensuring that air bubbles were not entrapped within the target, as the material was pluviated into the container. Transparent soils also required that RI mismatch be reduced by limiting the time involved in test setup, so as not to affect the temperature-sensitive RI of the saturating fluid. On balance, half space tests were easier to setup than quarter space tests.

\section{$2.4 \quad$ Optical Setup}

A NAC HX-5 high-speed digital camera was used for capturing images of the penetration events. Frame rates of 8,000, 10,000 and 10,200 frames per second (fps), were employed for capturing images of projectiles travelling at speeds of 60,100 , and $150 \mathrm{~m} / \mathrm{sec}$.

It was not possible to use LED lighting because it produced flicker that was visible in the captured images; thus, all tests were illuminated by 250 and 500-watt tungsten lamps. Tungsten lamps generate a significant amount of heat radiation, which can increase the temperature of the transparent targets, thus affecting transparency of the target, which depends on perfect matching of the temperature-sensitive RI of the saturating fluid and that of FQ. Accordingly, during testing, indirect lighting techniques were used to minimize this effect. In half space shots, the specimens were back lit by orienting the tungsten lamps to illuminate a white reflective surface behind the target, which provided silhouette images of projectile penetration. This technique resulted in uniform background brightness, which is a desired effect. All quarter space experiments were front lit by the use of the tungsten lamps oriented at approximately 45 degrees from the camera's line of sight to minimize glare and reflection of light into the camera.

In transparent specimens a linear laser displacement gage (LLDG) was used to record the depth of penetration (DOP) by measuring the distance between the original top of the soil and the final resting position of the projectile from the sides of the specimen un-intrusively, 
(fig. 4 inset). In opaque targets, the final DOP was recorded by taking measurements through the observation window, when possible, or by carefully exhuming the projectile.

\subsection{Scope}

A total of 11 tests were conducted in quarter space targets into FQ and Ottawa sand table 1. Measured initial speeds ranged from 65.9 to $158.8 \mathrm{~m} / \mathrm{s}$. Five tests were conducted on FQ specimens (W-1 to W-5) which yielded terminal DOP's in the range of $2.3 \mathrm{~cm}$ to $8.4 \mathrm{~cm}$. Six tests were conducted on Ottawa sand (W6 to W-11) which yielded terminal DOPs in the range of 3.1 to $8.3 \mathrm{~cm}$.

Seven tests were conducted in half space into FQ and Ottawa sand targets as listed in table 1. Measured initial speeds ranged from 65.9 to $154.8 \mathrm{~m} / \mathrm{s}$. Three tests were conducted in FQ specimens (M-1 to M-3), which yielded terminal DOP'S in the range of 3.2 to $8.7 \mathrm{~cm}$. Four tests were conducted in Ottawa sand (M-4 to M-7) which yielded terminal DOP's in the range of 4.5 to $6.5 \mathrm{~cm}$.

\section{OBSERVATIONS \& RESULTS}

\subsection{Shock wave and Compression Flash}

In quarter space experiments with dry soils, a detached bow shock was observed that traverses the opaque medium ahead of the projectile (fig. 6). The bow shock that is observed was not visible in transparent targets. An area of highly increased reflectivity is seen just ahead of the projectile in quarter space experiments into most of the opaque tests conducted, regardless of saturation, and in all of the transparent targets. This is believed to be caused by particle breakage ahead of the projectile, which would increase reflectivity, or by highly compressed arrangements of particles which would also increase reflectivity, or both. The increased area of reflectivity is referred to as "Compression Flash" and is about 1 to 1.5 projectile diameters in extent (fig. 7). Both observations were also noted by (Borg et al. 2013). Compression flash in opaque targets can only be observed at the beginning of penetration for 1 to 3 frames, during which time the projectile has penetrated typically about a couple of diameters. These observations were not visible in the half space experiments 
with transparent soils, perhaps due to the difference in the illumination schemes (front vs back illumination for quarter and half space, respectively). Additionally, the exhumed projectiles did not show signs of thermal damage, thus, it is unlikely that increased reflectivity can be attributed to frictional heating during penetration.

\subsection{Temporary and Permanent Loss of Transparency}

Videos of the penetration events into transparent soil at 60,100 , and $150 \mathrm{~m} / \mathrm{s}$ in half space are available for viewing (Video 1). Sequential snap shots of penetration into half and quarter space are presented herein for illustration in figure 8 to 13 . When looking at images of the penetration event in half space into a transparent soil surrogate, it is often hard to discern the location of the spherical projectile during penetration (fig. 8). The difficulty arises because of a loss of transparency within the crater and ahead of the projectile that result in an opaque region. Half space experiments are back-lit so images of the opaque region appear black (fig. 8). This opaque region was also observed in quarter space shots into sucrose saturated FQ (fig. 9). In these experiments the target was front-lit, and the area where transparency has been lost appears white. There are several hypotheses for the origin of this effect, including infusion of air into the granular medium from the cavity; dilatancy around the cavity and ahead of the projectile, or change in RI of the liquid due to changes in temperature or pressure. In all tests the opaque region regained transparency to some degree, shortly after the penetrator comes to rest, which was observed as a reduction in the size of the opaque zone. This observation is consistent with the hypothesis of dilatancycaused drop in saturation, followed by reentry of liquid into the opaque region.

\subsection{Compaction and Dilation Areas Ahead and Around the Projectile}

During penetration into quarter space sucrose saturated transparent FQ samples, after the initial compression flash subsides, two distinct regions can be distinguished. The first region is a less intense white zone ahead of the penetrator where we suspect compression flash is still occurring, but due only to compaction of particles ahead of the projectile. Compaction leads to a tighter packing of the particles which increases the reflection of light thus producing a visible white zone. The second region has much less reflectivity but lasted much 
longer (typically 2 to 3 milliseconds) (fig. $7 B$ ). Both of these regions are visible but not distinguishable in transparent half space experiments in which they appear as an opaque region due to the lighting setup. This phenomenon is not visible to that extent in dry materials. However, a "fuzzy" white outline of grains along the cavity along the shot-path can be seen in all dry targets (fig. 7A). The less reflective region in saturated materials during impact and penetration may be due to soil being dried out by dilatancy and subsequent cavitation. In general, the behavior is consistent with shear or hoop strain induced dilation as discussed above.

Due to its angular nature, granular FQ is a highly dilative material. When tightly packed and subjected to shear under low confining pressure, FQ particle arrangements will dilate. During penetration in order to make room for the fast-moving projectile, particles will rearrange out of their tightly packed structure increasing the bulk volume (dilation). In slow strain rate tests this occurs very slowly, and the pore fluid has time to fill up the newly created void spaces. During dynamic penetration, which is considered a high strain rate event, the area of dilation is visible because the soil structure dilates at a high speed rapidly forming new void spaces which cannot be filled up by the pore fluid at the same speed. In absence of pore fluid, the dilation area along with the newly created void spaces will then be filled with empty space or with air that has been introduced to the target during impact. Because empty space (RI 1.00000) and air (RI $=1.00029)$ have a different RI than transparent sucrose saturated FQ $(\mathrm{RI}=1.457)$, the mismatch in RI will inhibit transparency, and thus the dilation area becomes visible. This phenomenon is not visible in dry targets because they are not transparent to begin with.

At the end of penetration into transparent targets one of three things occurs; (1) If air is trapped within the dilation zone it becomes a permanent marker of the dilation area; (2) The newly created void spaces, due to dilation, will collapse and will not be visible; or (3) The newly created void spaces eventually get filled with pore fluid, thus becoming transparent again and not visible. The compaction and dilation areas can be observed in all of the transparent sucrose saturated FQ tests when shot in quarter space, W-1, W-2 and W-3 (fig. 9). This phenomenon was also observed in penetration events at similar speeds into half 
space transparent sucrose saturated FQ, M-1, M-2 and M-3 (fig. 8). However, in those tests because the targets were back lit, instead of increasing the visibility of the phenomenon the dilation area inhibits light from reaching the camera and an opaque region is created. During the event, this area reaches a maximum at some time after the projectile comes to rest and then retreats to some extent due to void collapse, or that the area becomes re-saturated. In the quarter space water saturated FQ test, W-4 (fig. 13), the visible compaction area is limited to the edges of the cavity and cavity healing mechanisms mentioned above are not visible.

\subsection{Shape of Crater}

The shape of the cavity is easily visible in quarter space. However, after the penetration event the cavity does not remain open due to ejecta falling back into the cavity and slumping, especially in dry conditions. Selected sequential images from high-speed video of quarter space tests in dry FQ and Ottawa sand are presented in figure 10 and figure 11. It was found that, for both materials, cavities in dry targets have the shape of a cone during the beginning of the penetration event which later takes the shape of a paraboloid as the projectile decelerates and comes to rest, ultimately followed by sand collapsing and filling up part of the cavity. This observation was consistent throughout the entire range of impact velocities employed in this study. Additionally, the observed crater shapes persisted despite rounded Ottawa sand having a smaller coefficient of internal friction than angular FQ. It should be noted that all specimens were tested without a top cover for the containers. Thus, during penetration a significant amount of ejecta ended up outside of the container, resulting in a loss of material, especially for quarter space tests.

Water saturated Ottawa sand cavities followed the same trend as dry targets, in quarter space (fig. 12) with the exception that the transition from a cone to a paraboloid takes more time to develop. Sucrose saturated FQ cavities (fig. 9) had a different shape than dry and water saturated Ottawa sand cavities. At lower speeds $(60 \mathrm{~m} / \mathrm{s})$ sucrose saturated FQ cavities had the shape of a pipe, which remained open after the penetration event. At higher speeds (100 to $150 \mathrm{~m} / \mathrm{s}$ ), the pipe transitions into a narrow cone which later transitions into a paraboloid in the $150 \mathrm{~m} / \mathrm{s}$ shot. At these speeds, the cavity also remains mainly open after 
the penetration event. To evaluate the effect of viscosity of the saturating fluid on the shape of the observed cavity, tests were also conducted in water saturated FQ (fig. 13). The observed crater was closer in shape to the crater observed in water saturated Ottawa sand than to sucrose saturated FQ. This suggests that viscosity of the saturating fluid has a significant effect on the shape of the open cavity, at least at the stress levels encountered in benchtop model tests. With the increase in viscosity comes a decrease in the size and shape of the cavity from an open cone to a narrow pipe.

A zone of white "fuzzy" sand is observed around the cavity in all opaque tests (fig. 10-13). This white sand is believed to be remnants of the Compression Flash resulting from particle breakage or comminution ahead of the projectile. The same material presumably exists in transparent quarter and half space tests (fig. 8 and 9), but is difficult to distinguish from entrapped air. The white zone of fractured particles decreases with time as the penetration event progresses, especially in dry tests where more soil mixing is believed to occur than in saturated tests.

After tests were complete, a permanent crater was visible in quarter space tests in transparent targets, and the projectile remained visible after the cavity was filled with the pore fluid that was displaced during the penetration event (fig. 14).

\subsection{Final Depth of Penetration (DOP)}

Locating the final depth of burial in our tests is easier said than done for two reasons. First, projectile rebound can lead to discrepancies between the observed final resting place of the projectile and the transient deepest penetration value. Projectile rebound is demonstrated in figure 15, where the projectile bounced after impact in dry and water saturated Ottawa sand targets. We have not been able to view rebound in the half space tests due to the aforementioned opacity surrounding the crater (fig. 8).

A second reason affecting the accuracy of determination of DOP is that when looking at video and individual images of penetration into opaque targets in quarter space, it is difficult to determine the projectile position after a few diameters of penetration. This is because as the projectile penetrates it slightly diverges from the observation window, and the cavity is 
quickly filled with granular particles or trapped air, or both, which inhibits the view of the projectile. This effect was more clearly visible in dry soils than in saturated soils. In most cases where the projectile was shot into a saturated granular medium it was found that the projectile traveled adjacent or close to the observation window. This was observed by either exhuming of the projectile or by observation through the transparent materials, viewing from the top or sides at the end of penetration (fig. 16A). However, when exhuming projectiles shot into dry Ottawa sand targets, it was found that the projectiles had deviated away from the observation window during penetration (fig. 16B). In these tests, it is assumed that the projectile ricochets and alters its trajectory due to a region of highly pressurized particles developing in between the projectile and the observation window which is responsible for deflecting the projectile and gouging of the window (fig. 17). The amount of deviation from the observation window at the end of penetration was recorded to be 2.9 and $1.3 \mathrm{~cm}$ for the $60 \mathrm{~m} / \mathrm{s}$ and $100 \mathrm{~m} / \mathrm{s}$ shots, respectively. The distance in the $150 \mathrm{~m} / \mathrm{s}$ shot could not be reliably recorded because of projectile rebound.

When projectile rebound occurs, the final value of DOP obtained through exhuming the projectile does not necessarily represent the deepest penetration of the projectile. However, by examining video photography of the cavity through the observation window, before the projectile in rebound is visible, we can deduce that the projectile penetrated at least one diameter below the deepest open cavity observed through the observation window before rebound is observed. This approximation yields the estimated maximum DOPs presented in table 1 for tests W-10 and W-11. Terminal DOP's of shots in quarter space and half space are presented in figure 18, where the initial velocity is plotted against the terminal DOP normalized by the diameter of the projectiles, which is a constant $(9.9 \mathrm{~mm})$. In Ottawa sand, terminal DOP in half space tests was equal or less than comparable quarter space tests. However, the terminal DOP was larger, by approximately 1D, for sucrose saturated FQ in half space tests than in quarter space. All quarter space tests in sucrose saturated FQ were shallower than dry or water saturated FQ.

When comparing terminal DOP of shots in quarter space of sucrose saturated FQ to data for sucrose saturated FQ samples shot in half space, it can be seen that they have comparable 
terminal DOP's (fig. 19 and table 2). This suggests that shooting in quarter space does not significantly affect terminal DOP. Terminal DOP of shots conducted in quarter space of Ottawa sand were compared to shots conducted in half space under similar conditions (saturation, gradation and relative density). There is excellent agreement in water saturated targets; in dry Ottawa sand targets, there is agreement in the shots with initial velocities of $60 \mathrm{~m} / \mathrm{s}$ and $100 \mathrm{~m} / \mathrm{s}$. However, the shot fired at an approximate speed of $150 \mathrm{~m} / \mathrm{s}$ in half space underperformed the shot conducted in quarter space. This is likely due to projectile rebound, which is unaccounted for in the shots made in half space because of opacity of the image during penetration.

The agreement between quarter space and half space test results for similar projectiles is somewhat surprising. The fact that these quarter space and half space measurements agree so well suggests that the sand between the projectile and the wall contributes nearly the same resistance to penetration as what would occur were the wall not there. Thus, the quarter space geometry not only provides a means to visualize in situ motion, it also provides a good estimate of penetration in a semi-infinite field.

As seen in the half space experiments, terminal DOP in quarter space increases with increasing initial velocity (Guzman et al. 2014). If we disregard the tests were rebound is either observed or suspected, we can also see that within this range of velocities that the relationship between initial velocity and terminal DOP is close to linear (fig. 18).

During penetration in quarter space, it has been sometimes difficult to distinguish the location of the bottom of the cavity during penetration. However, a frame-by-frame analysis of several of the tests listed in table 1 for quarter space yields enough data to construct a plot of depth of open cavity (as seen through the observation window) vs. time (fig. 20). The terminal DOP for each test is represented by a color dot. The transition of the bottom of the cavities, as seen through the observation window, is well behind the projectiles and did not correlate well with terminal DOP observations. Thus, shape of the cavity as seen through the observation window is not a good predictor of terminal DOP. However, apparent cavities provide insight that indicate when the terminal depth of penetration has been reached which most likely coincides with the moment that the cavity stops advancing. 
In an effort to validate the results of this study, terminal DOP data of sucrose saturated FQ in quarter space was compared to predicted terminal DOP values obtained using the Poncelet (1839) model, expressed as:

$P=\frac{1}{3} \frac{\rho_{p} d}{\rho_{t} C_{p}} \ln \left(\frac{\rho_{t} C_{p} v_{o}^{2}}{R}+1\right)$

Where, $\rho_{p}$ and $\rho_{t}$ are density of the projectile, and total (bulk) density of the target, respectively; and, $P$ and $v_{o}$ are the terminal DOP and impact velocity, respectively; $\mathrm{d}$ is projectile diameter; $C_{p}$ and $R$ are Drag coefficient and strength term proportional to the yield strength of the material, respectively, are used in Eq. (1).

Values recommended by Guzman, et. al. (2014) for sucrose saturated medium dense fused quartz, $\left(C_{p}=0.26\right.$ and $\left.\mathrm{R}=4 \mathrm{MPa}\right)$, along with $\rho_{p}$, and $\rho_{t},\left(7.60 \mathrm{~g} / \mathrm{cm}^{3}\right.$ and $1.83 \mathrm{~g} / \mathrm{cm}^{3}$, respectively), were used in combination with Poncelet's framework (Eq. 1) to predict terminal DOP. Measured terminal DOPs are plotted in Fig. 21 against the predicted terminal DOPs obtained using the Poncelet model. It can be seen that good predictions for shots in quarter space are obtained using this model.

\section{CONCLUSIONS}

Two techniques were compared in this study to visualize penetration mechanics into granular soils. Quarter space techniques which involve penetration against a rigid wall that acts as an observation window have long been employed as a useful tool to visualize granular mechanics in opaque soils. Additionally, recently developed transparent soils have been adapted in this study to also visualize projectile penetration into the middle of a target made of a transparent soil surrogate (half space). Both methods require different illumination setups. The use of a high-speed camera allowed us to observe and compare dynamic penetration into half space and quarter space. The following is a summary of how these two methods compare to each other, as well as the observations inferred from comparing penetration events in quarter and half space. 
- Quarter space method is an excellent tool for visualizing the projectile penetration event. Similar terminal DOP where found when comparing terminal DOP of shots into FQ and Ottawa sand in quarter space vs. shots in half space. Thus, it appears that shooting against an observation window does not significantly inhibit terminal DOP.

- Projectile rebound was observed when shooting at high speeds into quarter space dry specimens and water-saturated specimens. Therefore, the terminal DOP obtained by exhuming the target may not always be representative of the deepest penetration of the projectile, especially in half space experiments since rebound cannot be accurately accounted for using the methods presented herein.

- Test setup is much simpler, and less dangerous, in half space experiments than in quarter space experiments. In particular quarter space tests require much care in alignment to avoid contact between the top of the wall and the projectile which could ricochet into the open laboratory space. In contrast, if the projectile trajectory is too far away from the observation wall and into the soil, the event might not be visible through the observation window.

- Viscosity of the saturating fluid has a significant effect on the shape of the open cavity, with the increase in viscosity comes a decrease in the size and shape of the cavity. Shape of the cavity is not a good predictor of the terminal DOP.

- A detached bow shock and a zone of white sand was observed around the cavity in all opaque tests. This white sand is believed to be remnants of particle breakage or comminution ahead of the projectile. The same material presumably exists in transparent quarter and half space tests, although lighting setup thwarted the ability to identify it.

- A distinct dilation zone that travels ahead of a spherical projectile was observed in both scenarios, which inhibits transparency and view of the exact location of the projectile during penetration. Dilation zones in both scenarios had similar shape and size.

- In both methods, a certain degree of cavity healing occurs immediately after penetration, where the visible area of dilation is reduced. When cavitation due to dilation occurs during penetration the void either collapses after the event, gets 
permanently filled with trapped air or is eventually filled with pore fluid. After the dilation area has had time to heal, there is a permanent visible area that extends about 1.25 to 1.5 diameters ahead of the projectile.

\section{ACKNOWLEDGEMENT}

The authors gratefully acknowledge the support of the Defense Threat Reduction Agency (DTRA) Grant No: HDTRA1-10-1-0049. Fused quartz powder used in this investigation was manufactured by Mineral Technology Corporation (Mintec). Low Color Sucrose ${ }^{\mathrm{TM}}$ used to match the granular fused quartz was manufactured by Indiana Sugars. The HX-5 high-speed camera was manufactured by NAC Image Technology Inc.

\section{SUPPLEMENTARY MATERIAL}

Video 1 - Observations of Projectile Penetration into Transparent Soils 


\section{REFERENCES}

Borg, J.P., M. Morrissey, C. Perich, T. Vogler, and L. Chhabildas. 2013. "In situ velocity and stress characterization of a projectile penetrating a sand target: Experimental measurements and continuum simulations." International Journal of Impact Engineering 51, (January): 23-35. https://doi.org/10.1016/j.ijimpeng.2012.07.009.

Borg, J.P., and J.T. Vogler. "An Experimental Investigation of a High Velocity Projectile Penetrating Sand." XIth International Congress and Exposition, Orlando, Florida, USA, June, 2008.

Casagrande, A. 1936. "Characteristics of cohesionless soils affecting the stability of slopes and earth fills." Journal of the Boston Society of Civil Engineers 23:13-32.

Cave, A., S. Roslyakov, M. Iskander, and S. Bless. 2014. "Design and performance of a laboratory pneumatic gun for soil ballistic applications." Experimental Techniques, Society of Experimental Techniques, (April). https://doi.org/10.1111/ext.12091.

Chen, Zhibo, Mehdi Omidvar, Magued Iskander, and Stephan Bless. 2014. "Modelling of projectile penetration using transparent soils." International Journal of Physical Modelling in Geotechnics 14, no. 3 (May): 68-79. https://doi.org/10.1680/ijpmg.14.00003

Ezzein, F. M, and R. J. Bathurst. 2011. "A transparent sand for geotechnical laboratory modeling." ASTM Geotechnical Testing Journal 34, no. 6 (November):590-601. https://doi.org/10.1520/gtj103808

Ferreira, J., and J. Zornberg. 2015. "A Transparent Pullout Testing Device for 3D Evaluation of Soil-Geogrid Interaction." Geotechnical Testing Journal 38 (25):686-707. doi: 10.1520/GTJ20140198. https://doi.org/10.1520/gti20140198

Guzman, I.L., and M. Iskander. 2013. "Geotechnical properties of sucrose-saturated fused quartz for use in physical modeling." Geotechnical Testing Journal 36, no. 3 (May):448454. https://doi.org/10.1520/gtj20120182

Guzman, I.L., M. Iskander, S. Bless, and C. Qi. 2014a. "Terminal depth of penetration of spherical projectiles in transparent granular media." Granular Matter 16, no. 6 (October):829-842. https://doi.org/10.1007/s10035-014-0528-y

Guzman, I.L., M. Iskander, E. Suescun-Florez, and M. Omidvar. 2013. "A transparent aqueoussaturated sand surrogate for use in physical modeling." Acta Geotechnica 9, no. 2 (April):187-206. https://doi.org/10.1007/s11440-013-0247-2

Guzman, Ivan L., Magued Iskander, and Stephan Bless. 2015. "Observations of projectile penetration into a transparent soil." Mechanics Research Communications 70, (December):4-11. https://doi.org/10.1016/j.mechrescom.2015.08.008

Holtz, R.D., W.D. Kovaks, and Sheahan T.C. 2010. An Introduction to Geotechnical Engineering, 2nd Edition. 2nd ed: Prentice Hall.

Iskander, M. 2010. Modelling with Transparent Soils, Visualizing Soil Structure Interaction and Multi Phase Flow, Non-Intrusively, Geomechanics \& Geoengineering: Springer.

Iskander, Magued. 2016. "Transparent Soil Wiki." https://perma.cc/66GC-C7F2.

Iskander, Magued, Stephen Bless, and Mehdi Omidvar. 2015. Rapid penetration into granular media: visualizing the fundamental physics of rapid earth penetration: Elsevier. 
Omidvar, M., J. Doreau Malioche, Z. Chen, M. Iskander, and S. Bless. 2015. "Visualizing Kinematics of Dynamic Penetration in Granular Media Using Transparent Soils." Geotechnical Testing Journal 38, no. 5 (September):656-672. https://doi.org/10.1520/gtj20140206

Omidvar, M., M. Iskander, and S. Bless. 2012. "Stress-strain behavior of sand at high strain rates." International Journal of Impact Engineering 49, (November):192-213. https://doi.org/10.1016/j.ijimpeng.2012.03.004

Omidvar, M., M. Iskander, and S. Bless. 2014. "Response of granular media to rapid penetration." International Journal of Impact Engineering 66, (April):60-82. https://doi.org/10.1016/j.ijimpeng.2013.12.004

Piekutowski, A.J. "Formation of bowl-shaped craters." Eleventh Lunar Planetary Science Conference, Houston, Texas, March,1980.

Poncelet, J.V. 1839. Introduction li la Mecanique Industrielle. Second Edition ed. Brussels.

Powers, M.C. 1953. "A new roundness scale for sedimentary particles." Journal of Sedimentary Research 23, no. 2 (June):117-119. https://doi.org/10.1306/D4269567-2B26-11D78648000102C1865D 
Table 1 Summary of all tests performed in this study

\begin{tabular}{|c|c|c|c|c|c|c|c|}
\hline Test & $\begin{array}{l}\text { Granular } \\
\text { Material }\end{array}$ & $\begin{array}{c}\text { Saturation } \\
\text { type }\end{array}$ & $\begin{array}{l}\text { Relative } \\
\text { Density }\end{array}$ & $\begin{array}{c}\text { Dry } \\
\text { Density, } \\
\text { g/cm } \\
\end{array}$ & $\begin{array}{c}\text { Total } \\
\text { Density, } \\
\text { g/cm } \\
\end{array}$ & $\begin{array}{c}\text { Initial } \\
\text { Velocity, } \\
\text { m/s }\end{array}$ & $\begin{array}{c}\text { Depth of } \\
\text { Penetration, } \\
\mathrm{cm}\end{array}$ \\
\hline W-1 & FQ $(-20+60)$ & Sucrose & 60 & 1.18 & 1.80 & 65.9 & 2.3 \\
\hline $\mathrm{W}-2$ & FQ $(-20+60)$ & Sucrose & 60 & 1.18 & 1.80 & 107.5 & 4.3 \\
\hline W-3 & FQ $(-20+60)$ & Sucrose & 60 & 1.18 & 1.80 & 157.2 & 6.2 \\
\hline$W-4$ & FQ $(-20+60)$ & Water & 65 & 1.17 & 1.64 & 148.4 & 7.7 \\
\hline$W-5$ a & FQ $(-20+60)$ & Dry & 59 & 1.16 & 1.16 & 158.8 & 8.4 \\
\hline W-6 & Ottawa Sand & Dry & 64 & 1.77 & 1.77 & 66.7 & 4.3 \\
\hline W-7 & Ottawa Sand & Dry & 68 & 1.79 & 1.79 & 99.3 & 5.9 \\
\hline$W-8^{a}$ & Ottawa Sand & Dry & 63 & 1.77 & 1.77 & 150 & 8.3 \\
\hline W-9 & Ottawa Sand & Water & 58 & 1.75 & 2.09 & 66.6 & 3.1 \\
\hline$W-10^{a}$ & Ottawa Sand & Water & 62 & 1.77 & 2.10 & 105.8 & 4.5 \\
\hline $\mathrm{W}-11^{\mathrm{a}}$ & Ottawa Sand & Water & 65 & 1.77 & 2.10 & 147.5 & 6.0 \\
\hline$M-1 b$ & $\mathrm{FQ}(-20+60)$ & Sucrose & 65 & 1.18 & 1.80 & 66.3 & 3.2 \\
\hline$M-2 b$ & FQ $(-20+60)$ & Sucrose & 69 & 1.19 & 1.80 & 104.6 & 4.8 \\
\hline$M-3^{b}$ & FQ $(-20+60)$ & Sucrose & 65 & 1.18 & 1.80 & 149.5 & 8.7 \\
\hline$M-4$ & Ottawa Sand & Dry & 65 & 1.77 & 1.77 & 65.9 & 4.5 \\
\hline M-5 & Ottawa Sand & Dry & 61 & 1.76 & 1.76 & 105.5 & 5.1 \\
\hline M-6 & Ottawa Sand & Dry & 64 & 1.77 & 1.77 & 154.8 & 6.5 \\
\hline M-7 & Ottawa Sand & Water & 63 & 1.77 & 2.1 & 147.1 & 5.8 \\
\hline
\end{tabular}


Table 2 Comparison of terminal DOP of shots in quarter space and shots into half space

\begin{tabular}{ccccc}
\hline \hline $\begin{array}{c}\text { Granular } \\
\text { Material }\end{array}$ & $\begin{array}{c}\text { Saturation } \\
\text { Condition }\end{array}$ & $\begin{array}{c}\text { Nominal } \\
\text { Initial } \\
\text { Velocity, } \\
\mathbf{m} / \mathbf{s}\end{array}$ & $\begin{array}{c}\text { DOP } \\
\text { Quarter Space, } \\
\mathbf{c m}\end{array}$ & $\begin{array}{c}\text { DOP } \\
\text { Half Space, } \\
\mathbf{c m}\end{array}$ \\
\hline \hline $\begin{array}{c}\text { Fused } \\
\text { Quartz }\end{array}$ & Sucrose & 60 & 2.3 & $3.2^{\mathrm{a}}$ \\
& Sucrose & 100 & 4.3 & $4.8^{\mathrm{a}}$ \\
& Sucrose & 150 & 6.2 & $8.7^{\mathrm{a}}$ \\
\hline \hline Ottawa Sand & Dry & 60 & 4.3 & $4.5^{2}$ \\
& Dry & 100 & 5.9 & 5.1 \\
& Dry & 150 & $8.3 \mathrm{~b}$ & $6.5^{\mathrm{c}}$ \\
& Water & 150 & 6.0 & $5.8^{\mathrm{b}}$ \\
\hline \hline
\end{tabular}

a From (Guzman, Iskander, and Bless 2015)

b Because of projectile rebound approximated from observations through observation window of maximum cavity depth

c Projectile rebound likely, but cannot be observed in this type of testing 


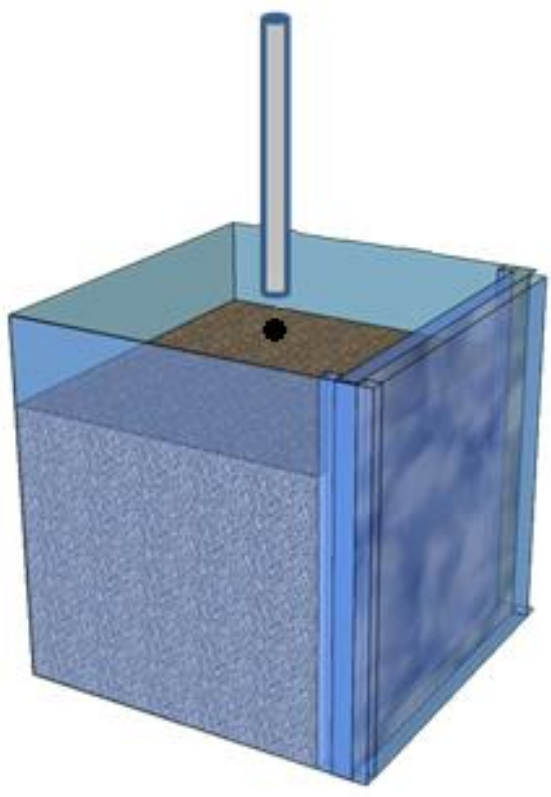

HALF SPACE

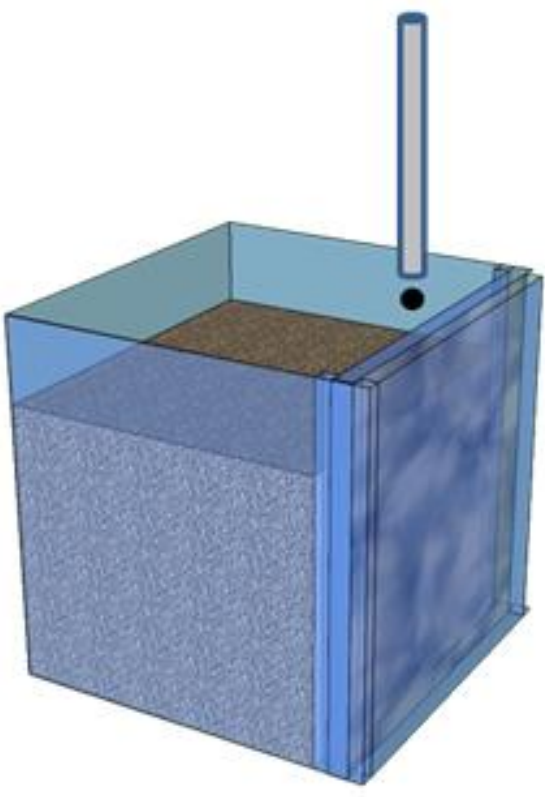

QUARTER SPACE

Figure 1 Schematic drawing of half space (left) and quarter space (right) setups. 


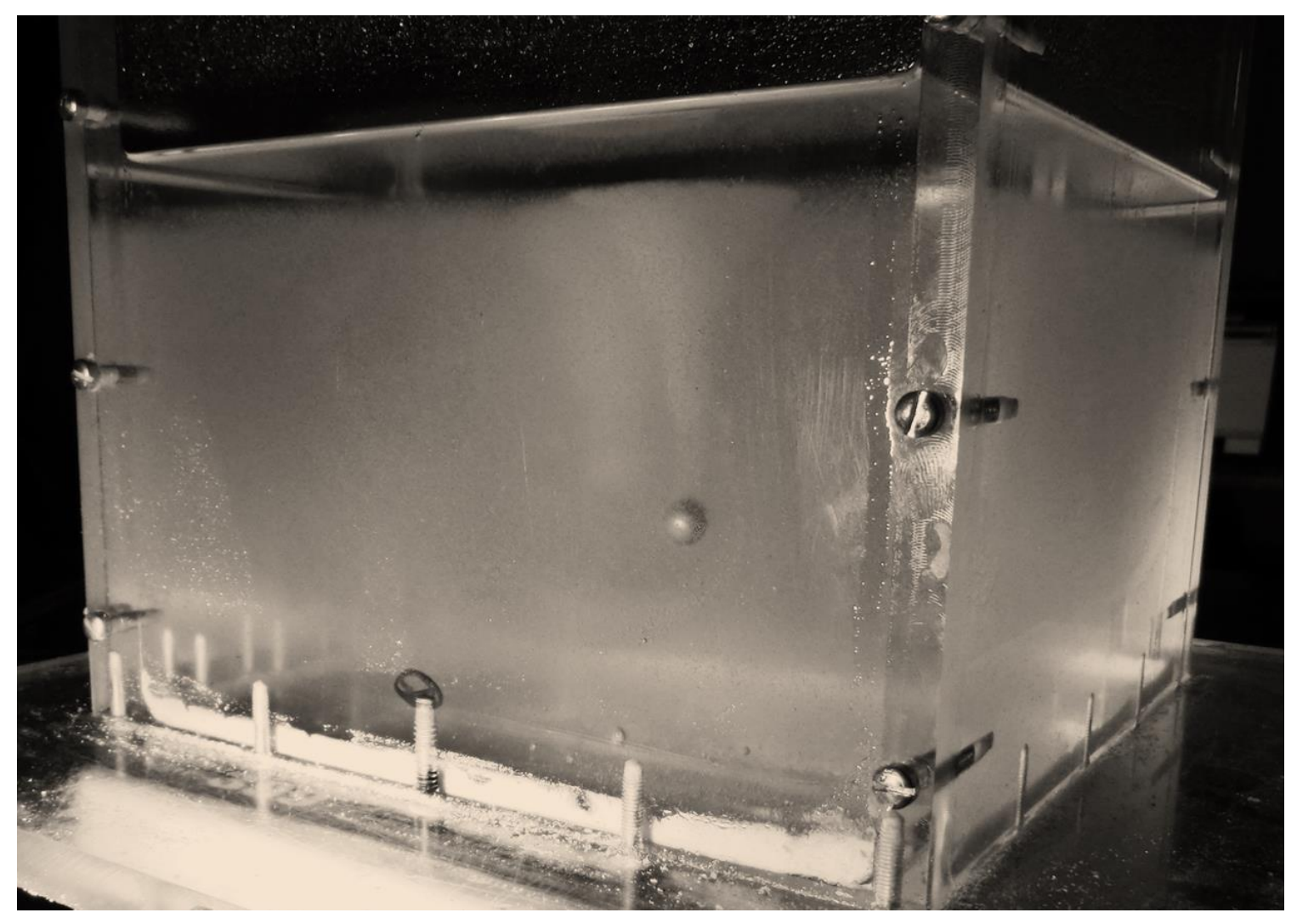

Figure 2 Projectile at rest as seen through transparent soil. 

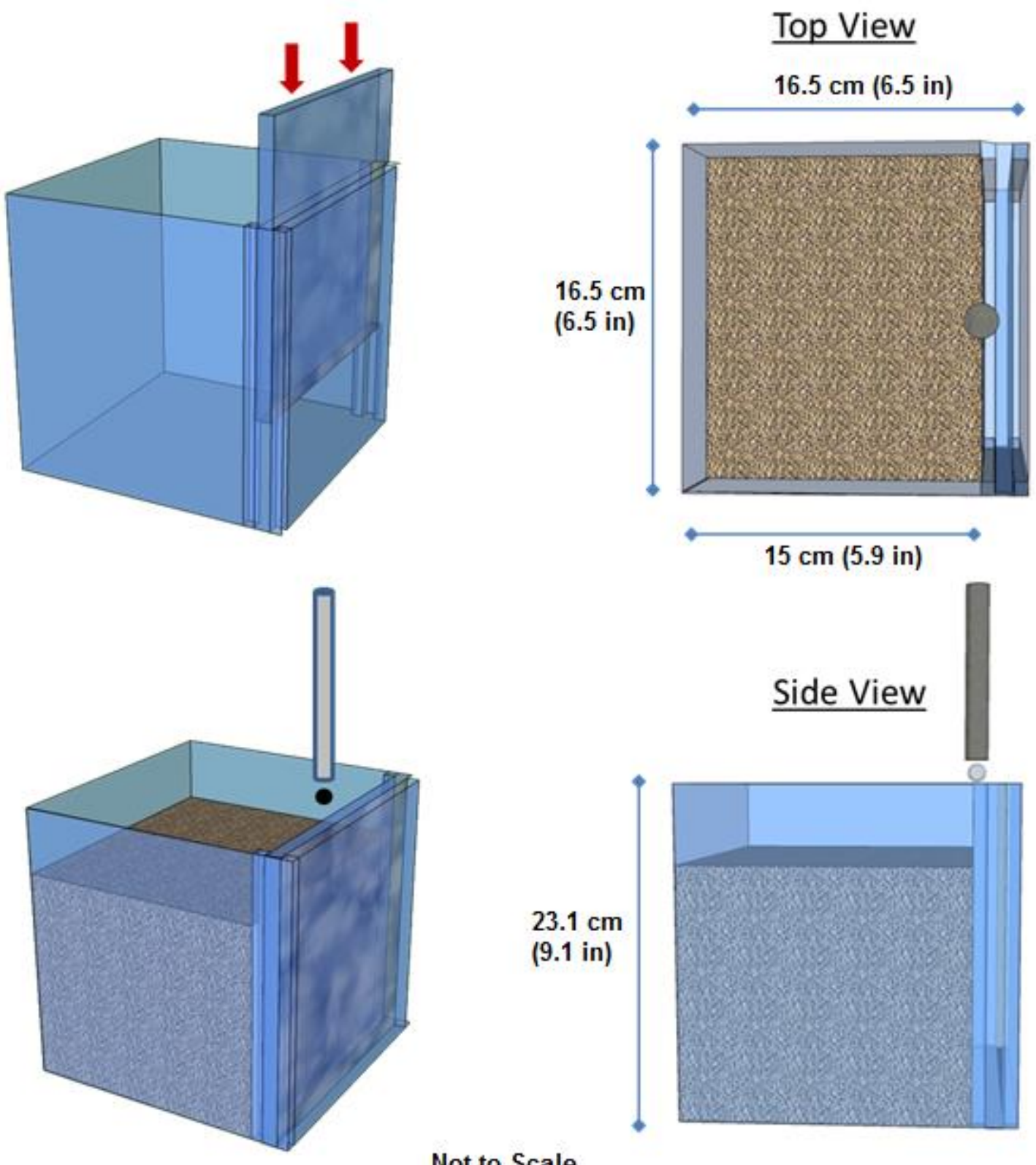

Not to Scale

Figure 3 Sketches of acrylic container with removable wall used for penetration in quarter space study. Installation of removable wall (top left), top view (top right). Shooting of a projectile against the removable wall (bottom left and right) 


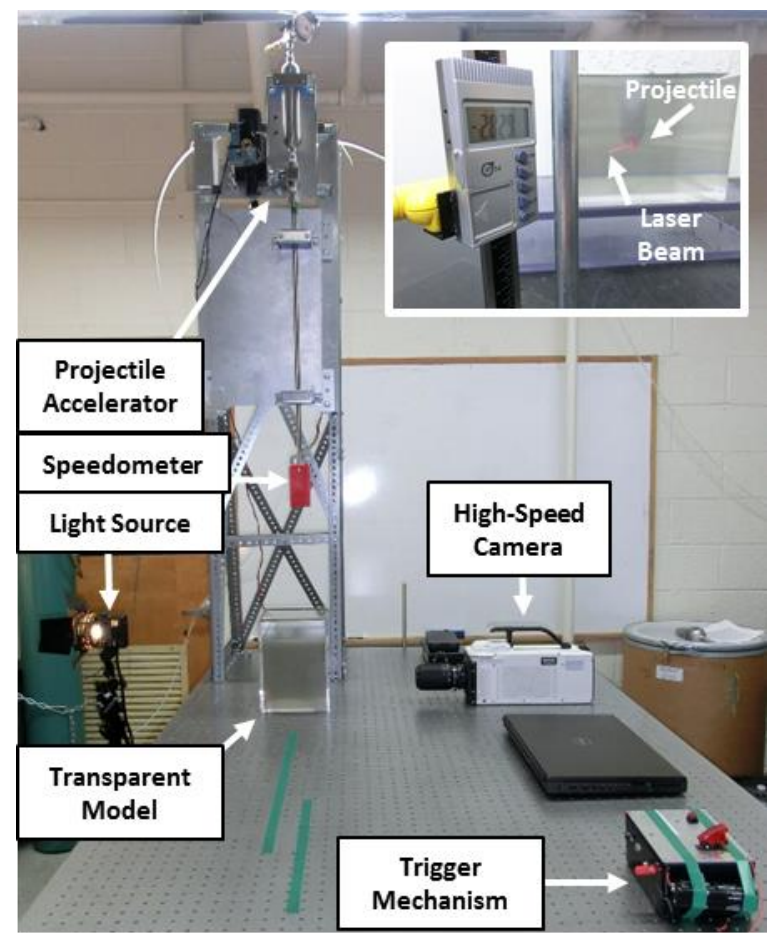

Figure 4 Experimental setup and LLDG (inset) used to measure final depth of penetration of projectile after impact. 


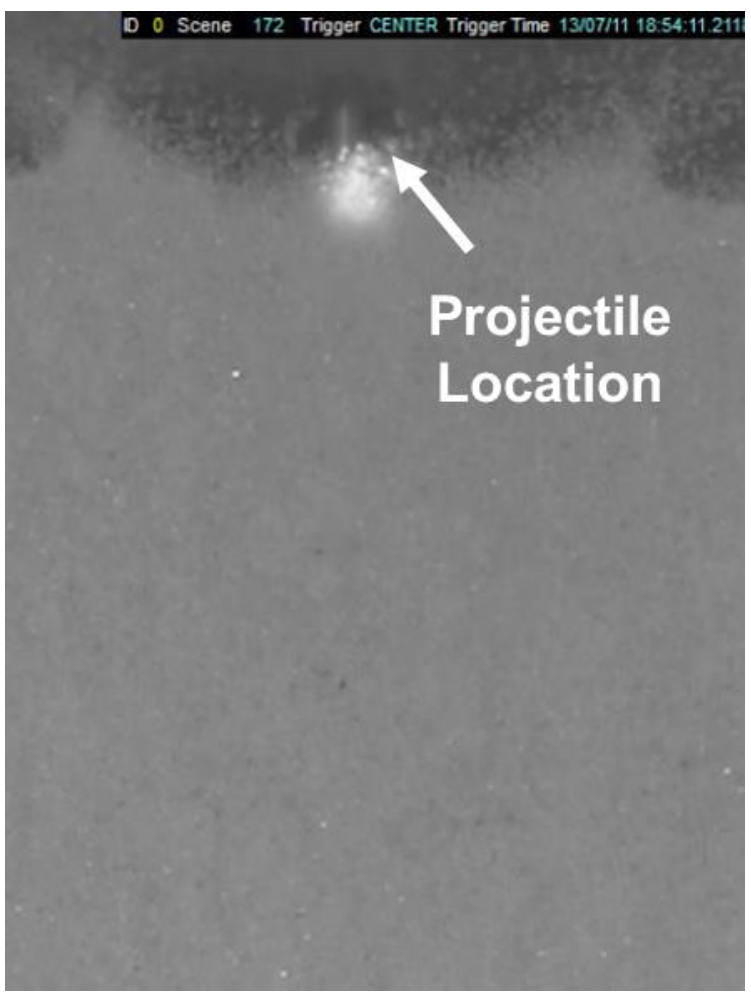

Figure 5 Projectile at impact into quarter space dry Ottawa sand at $150 \mathrm{~m} / \mathrm{s}$ (Test W-8). 


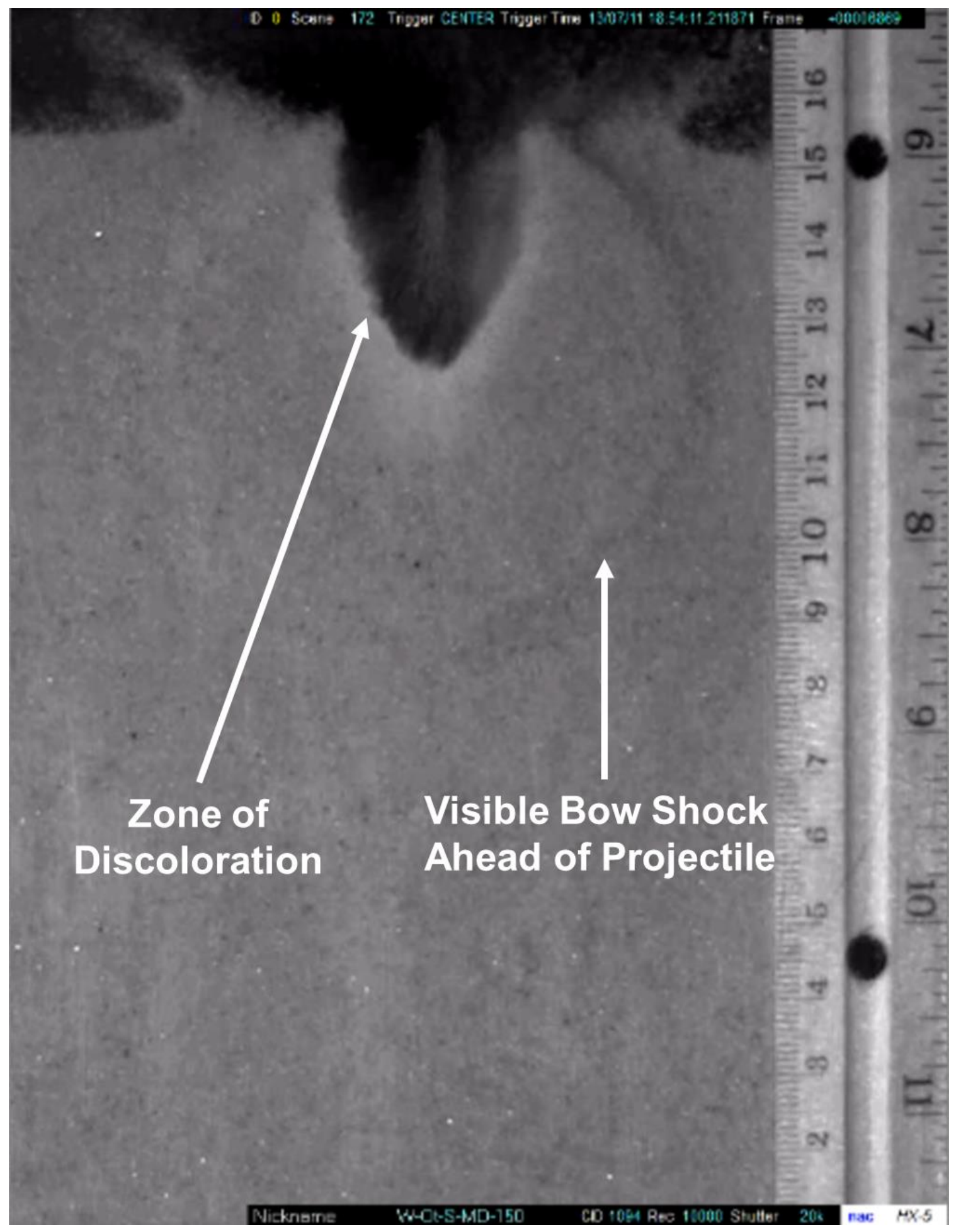

Figure 6 Example of visible bow shock and particle crushing leading to discoloration of sand in cavity in quarter space experiments into dry Ottawa Sand (Test W-8). 

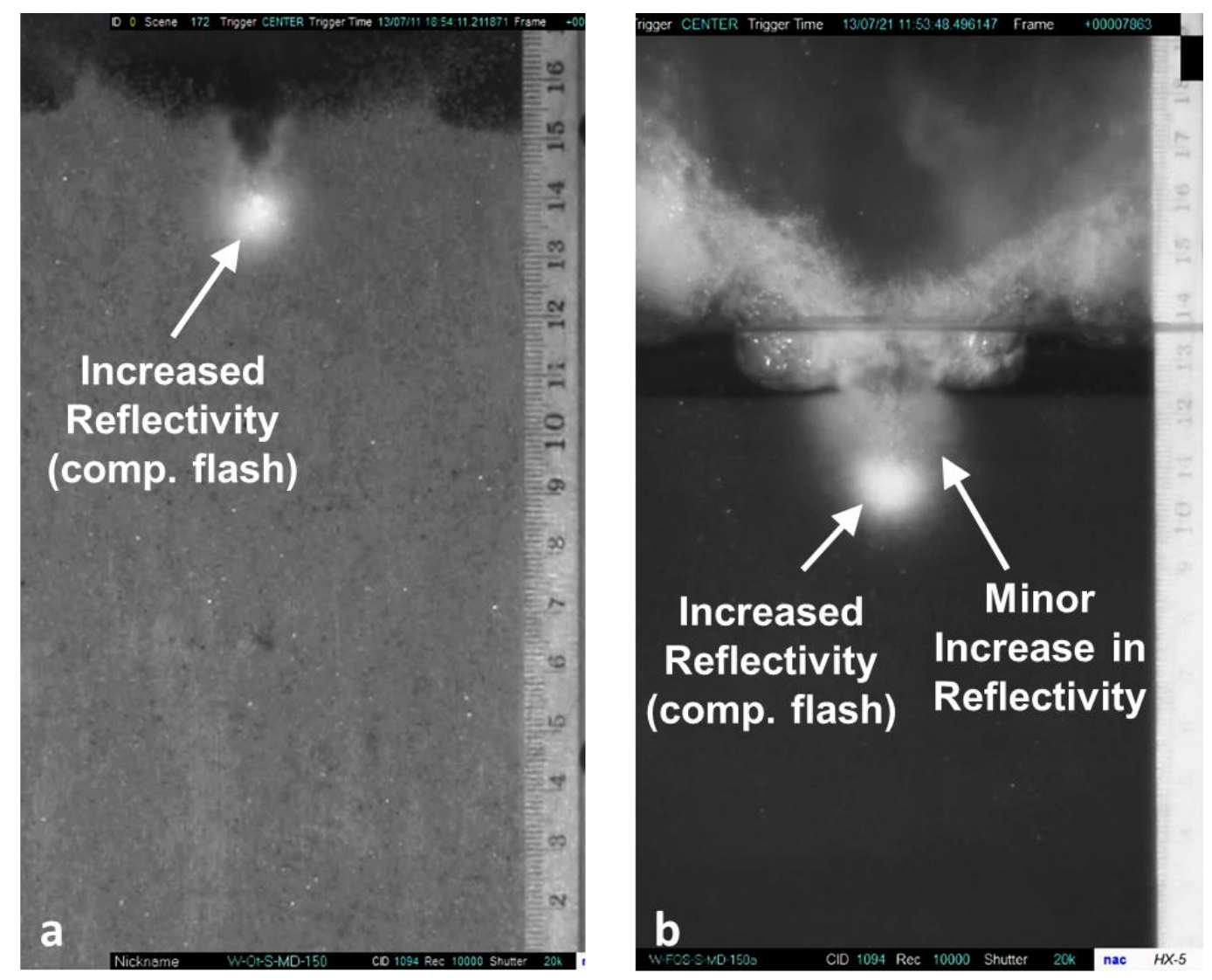

Figure 7 Compression flash at projectile tip; (A) in an opaque granular medium (Test $\mathrm{W}-8$ ), and (B) in a transparent granular medium (Test W-3), during quarter space penetration. Both scenarios are front lit 

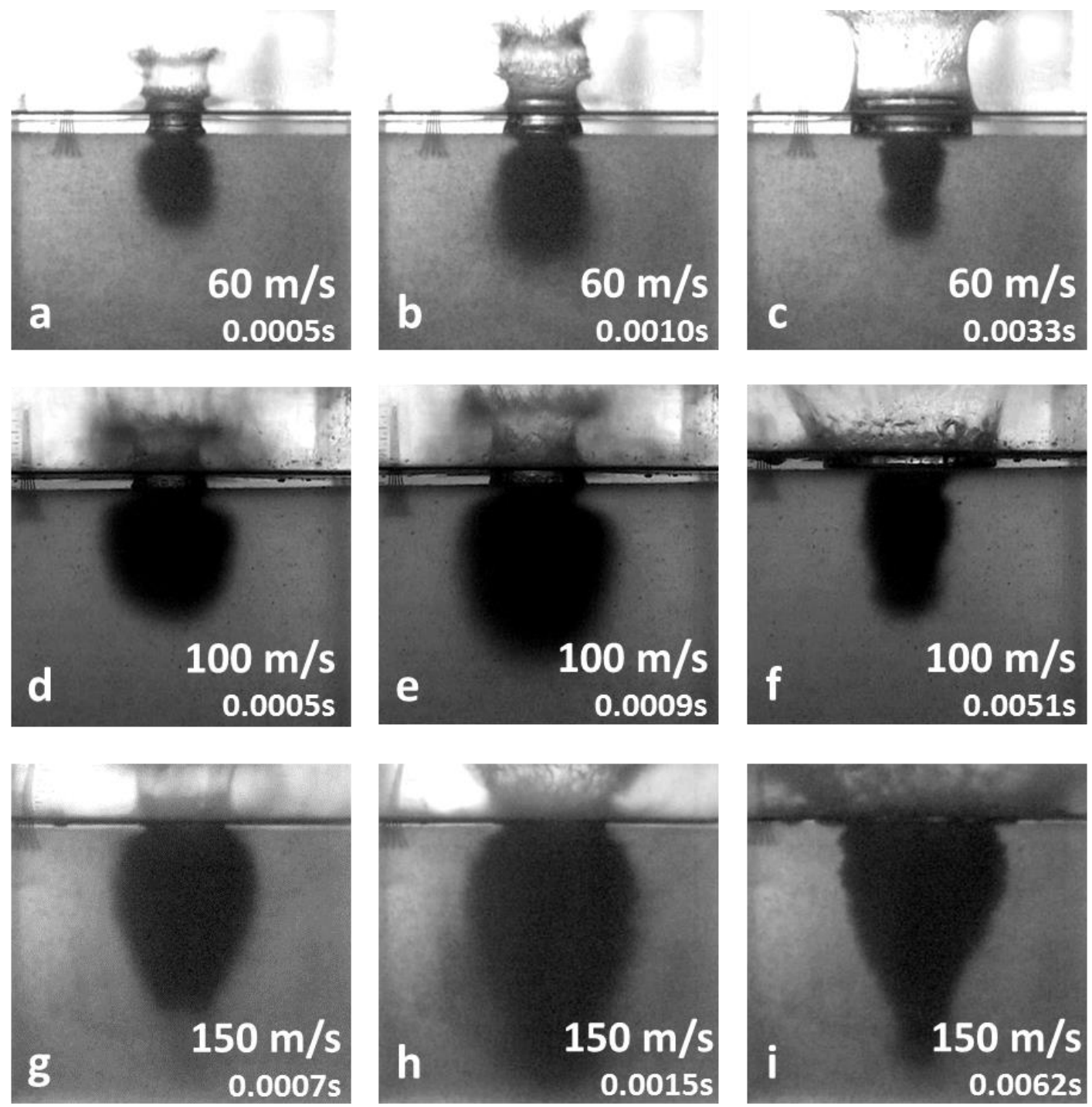

Figure 8 Progressive snapshots of penetration in half space (Sucrose Saturated FQ). Tests M-1 (top row), M-2 (middle row) and M-3 (bottom row) 

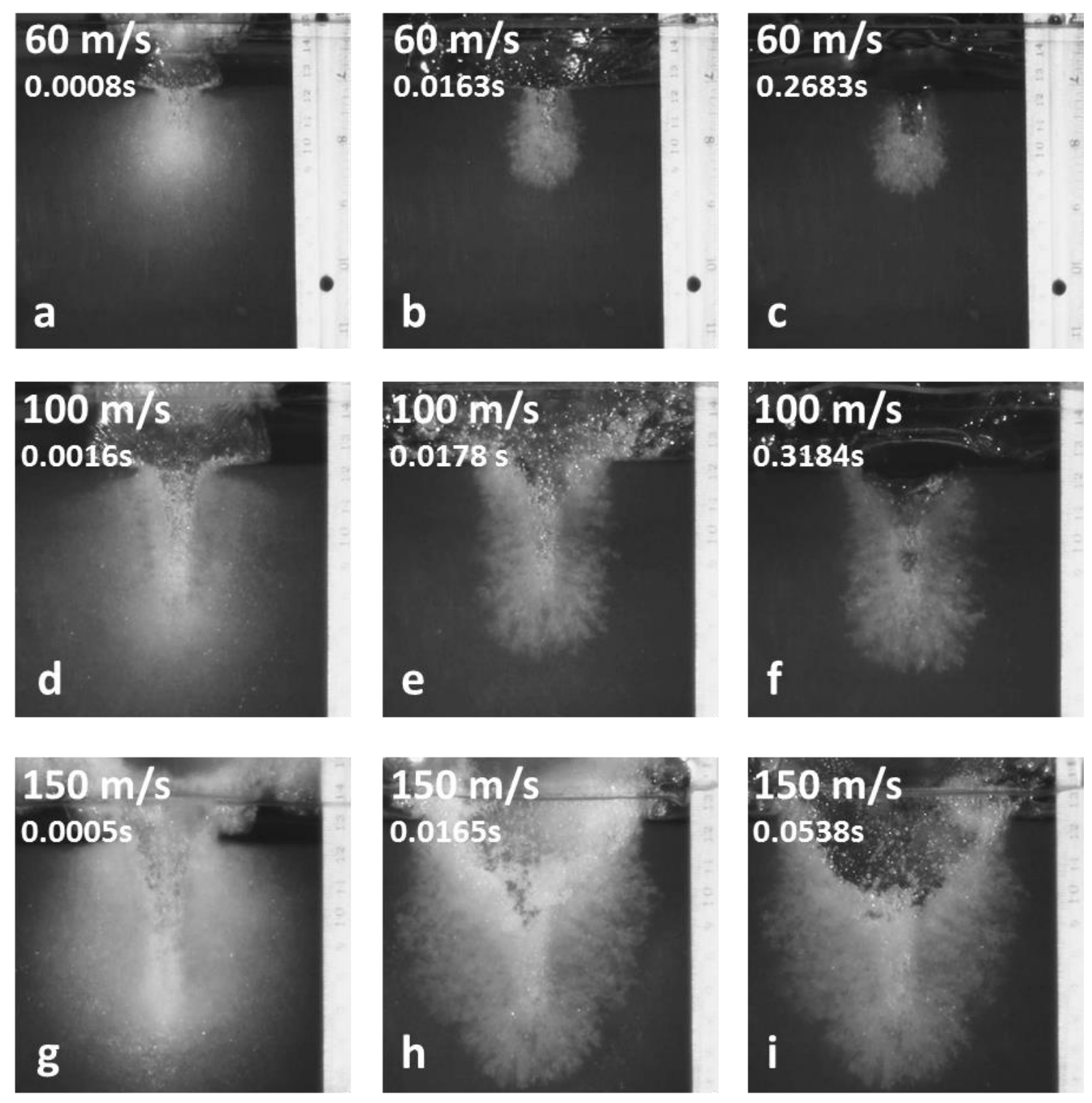

Figure 9 Progressive snapshots of penetration events in quarter space (Sucrose Saturated FQ). Tests W-1 (top row), W-2 (middle row) and W-3 (bottom row) 

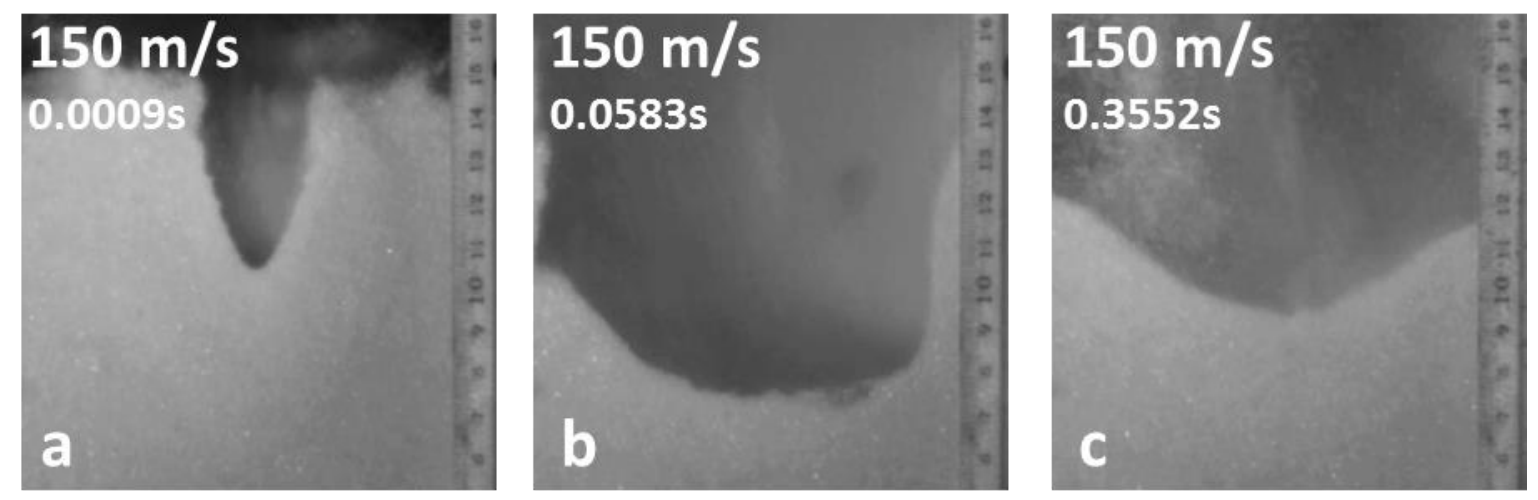

Figure 10 Progressive snapshots of penetration in quarter space (Dry FQ). Test W-5 

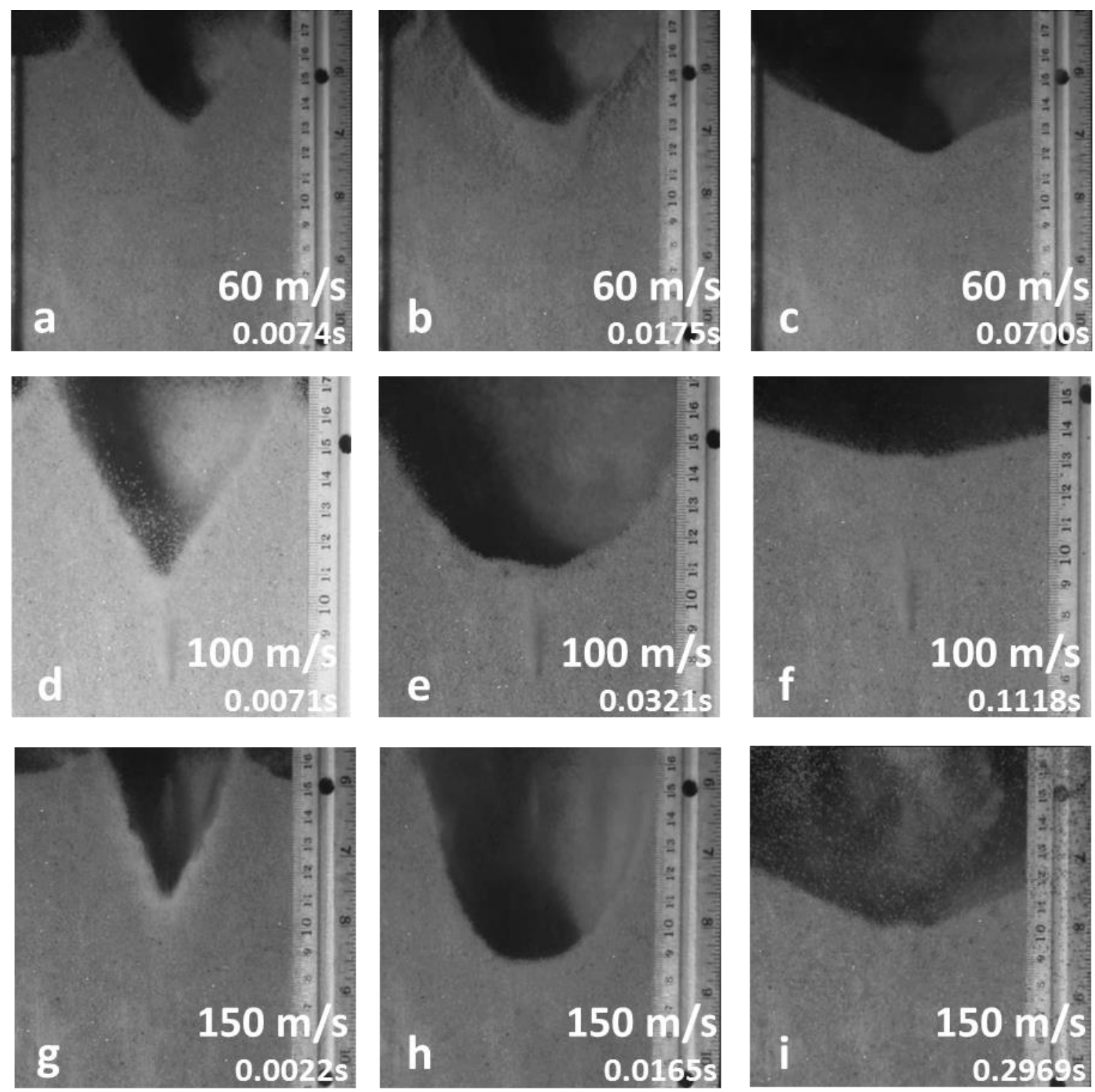

Figure 11 Progressive snapshots of penetration in quarter space (Dry Ottawa Sand). Tests W-6 (top row), W-7 (middle row) and W-8 (bottom row) 

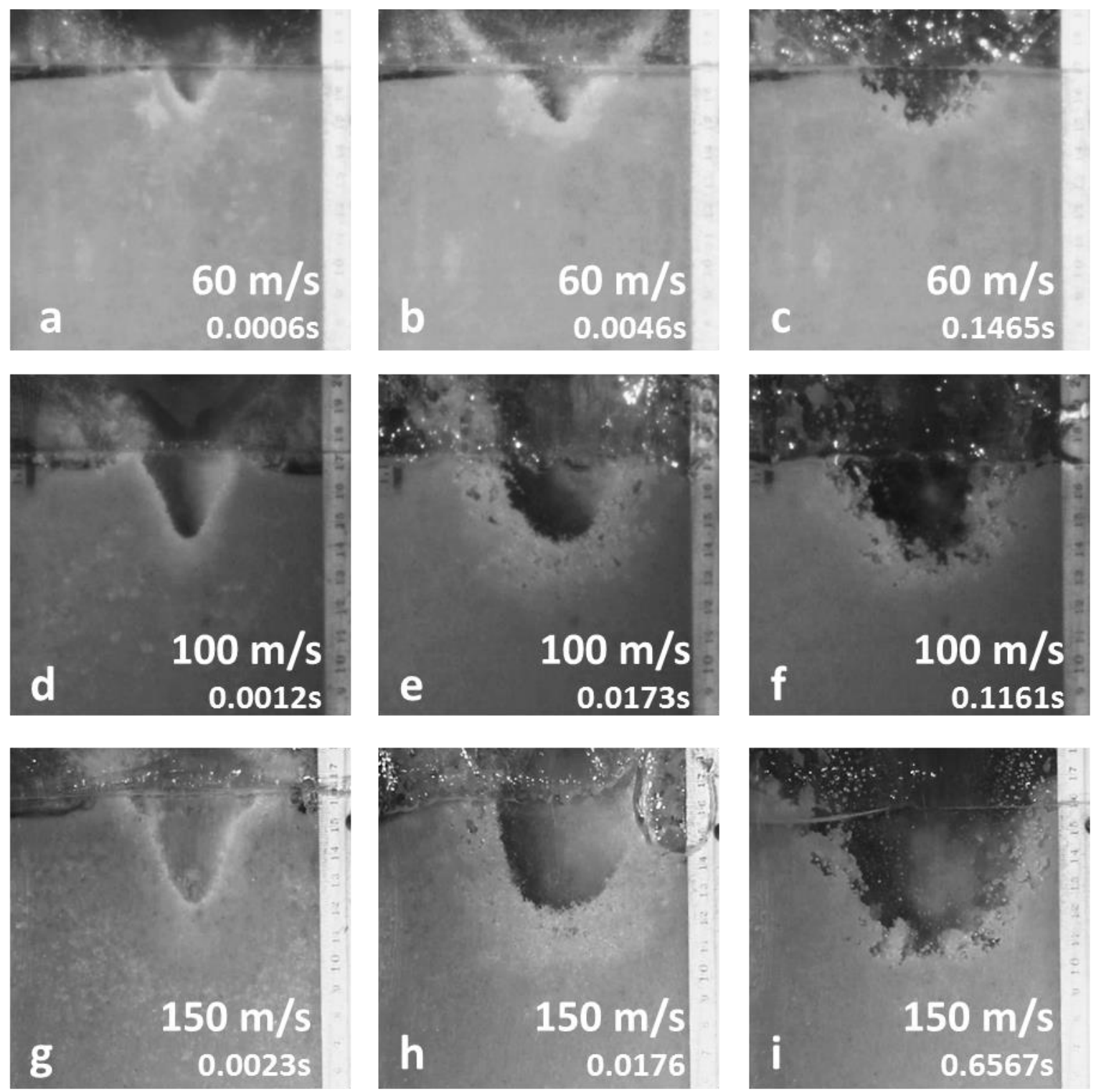

Figure 12 Progressive snapshots of penetration in quarter space (Water saturated Ottawa sand). Tests W-9 (top row), W-10 (middle row) and W-11 (bottom row) 

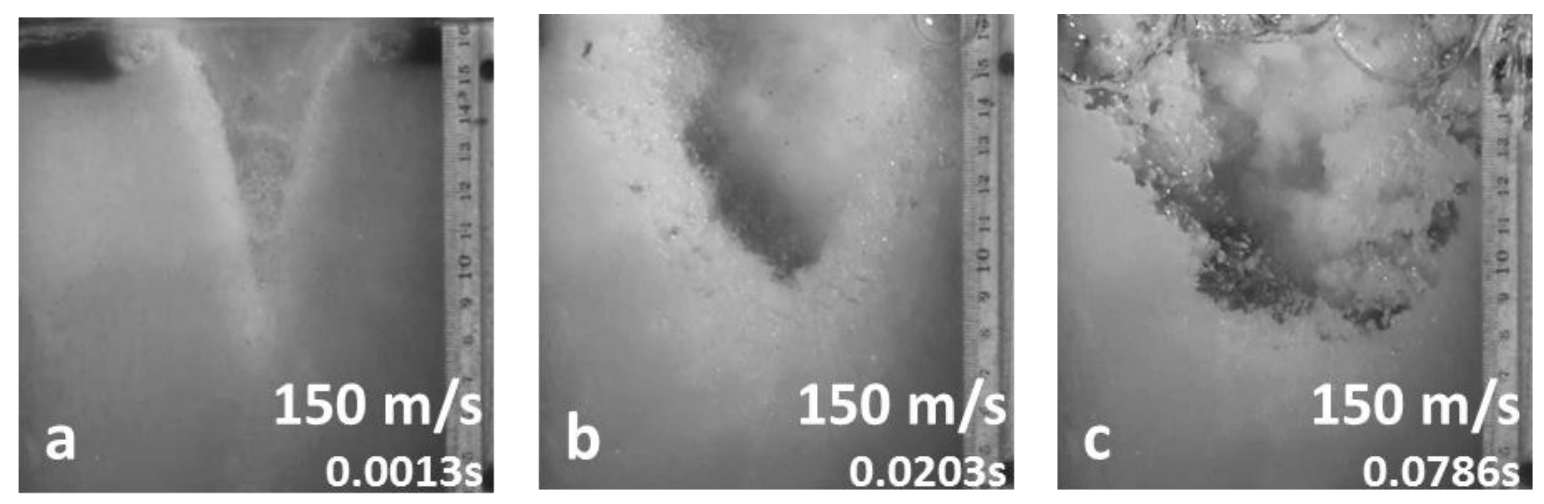

Figure 13 Progressive snapshots of penetration in quarter space (Water Saturated FQ). Test W-4 

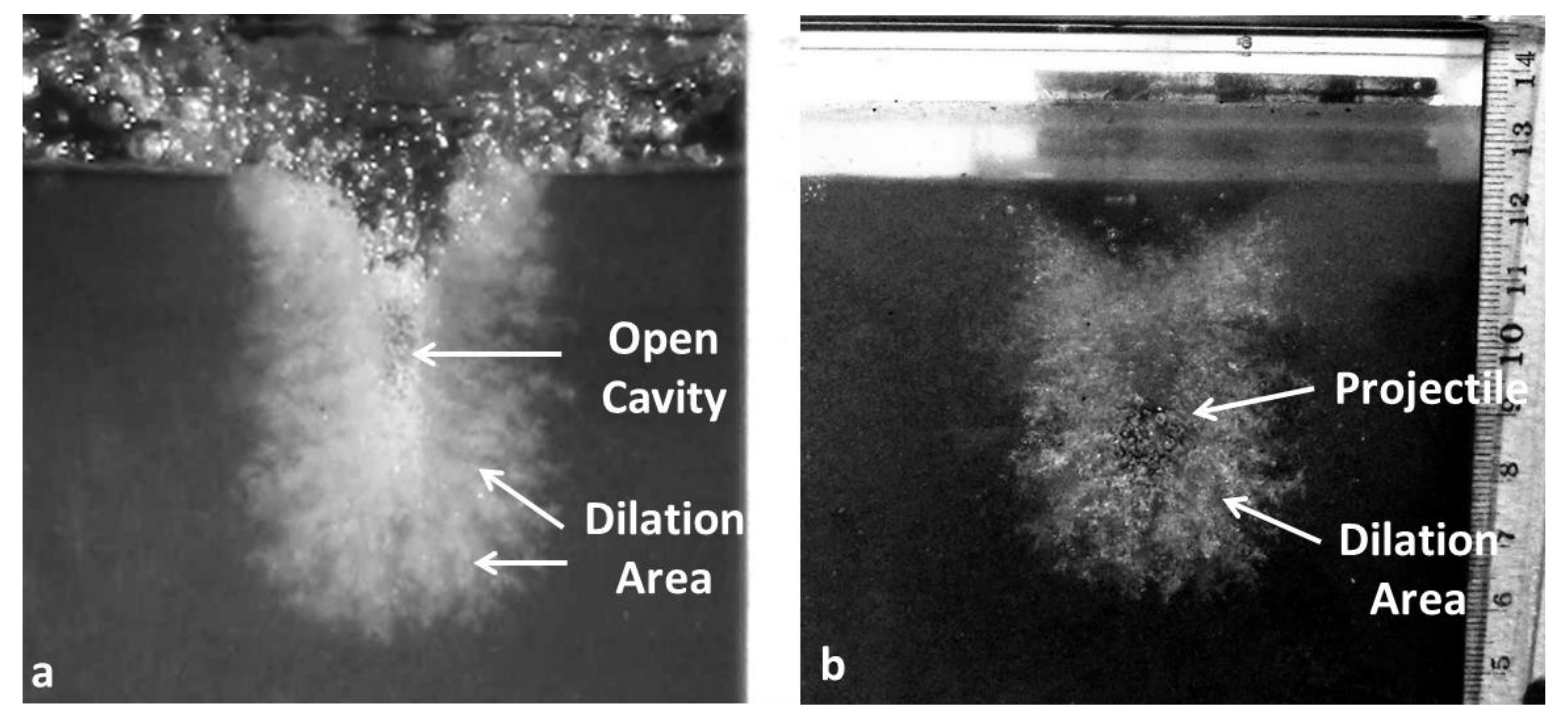

Figure 14 Images of test in quarter space into sucrose-saturated fused quartz (W-2). (A) Snapshot from high-speed camera near the end of penetration. (B) Picture at the end of same penetration event after the cavity has partially healed. 

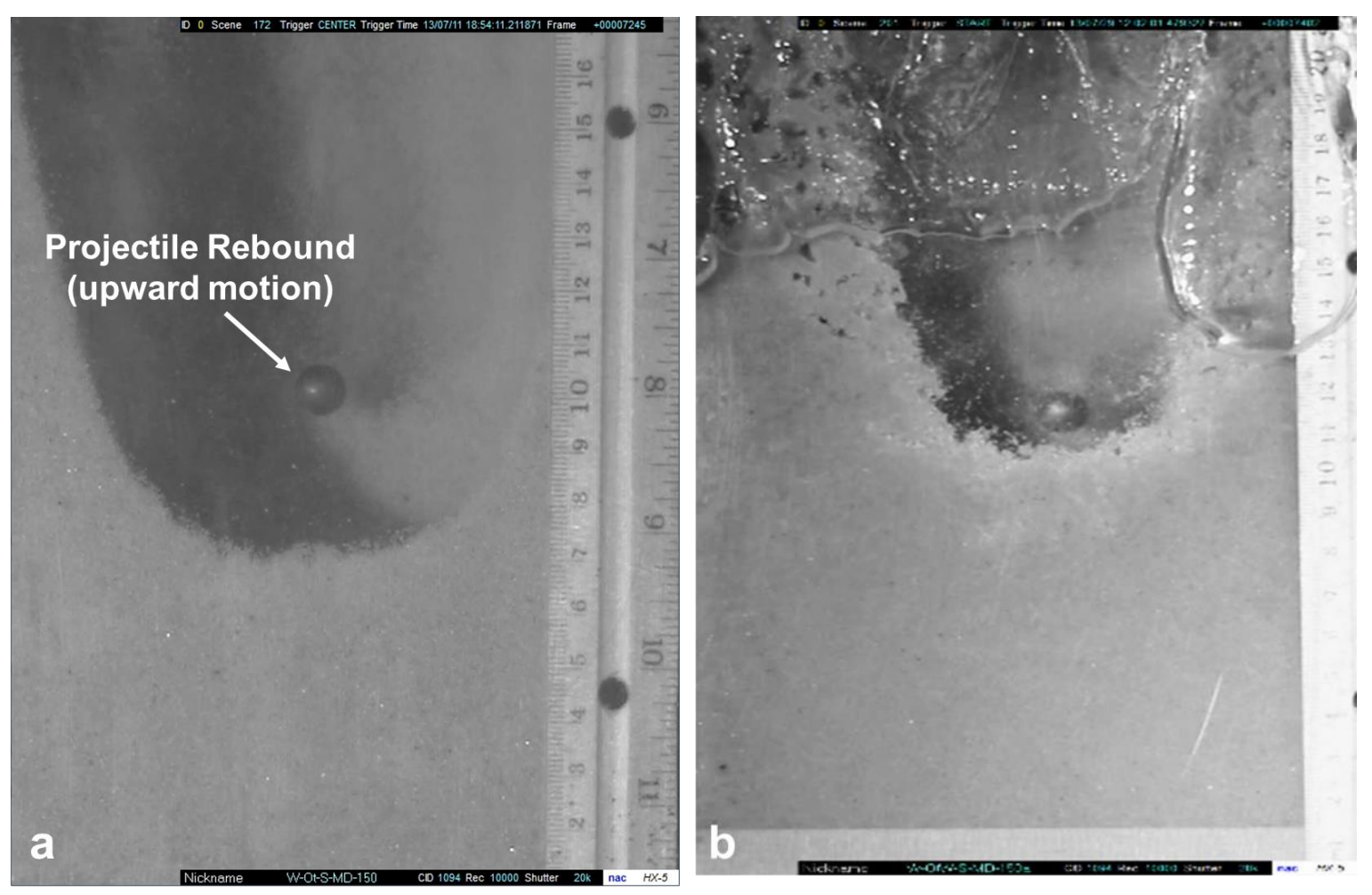

Figure 15 Projectile rebound observed in quarter space tests; (A) W-8 (Ottawa Sand Dry) and; (B) W-11 (Ottawa Sand - Water Saturated). 

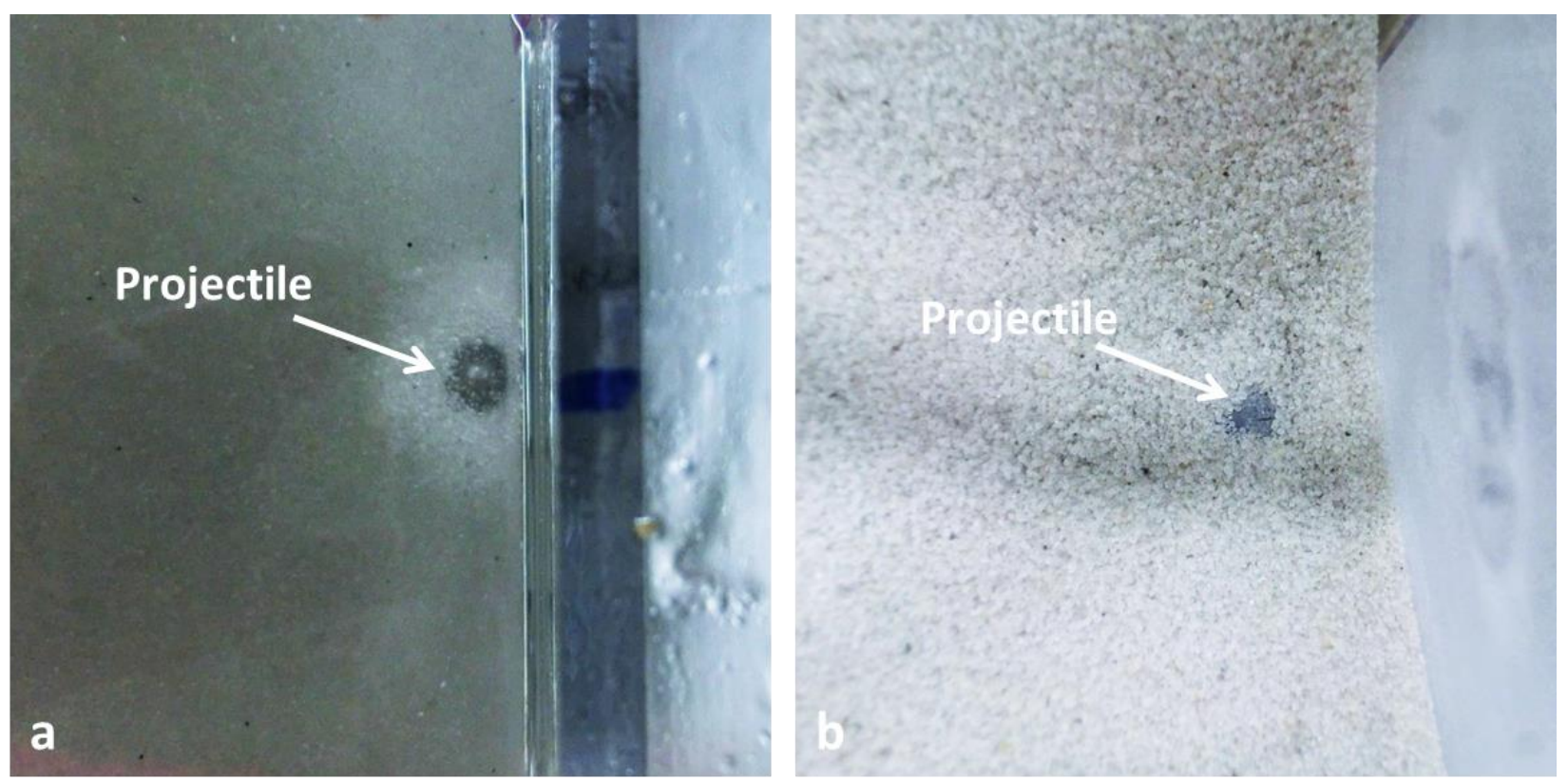

Figure 16 Top view of projectiles shot in quarter space after the penetration event into; (A) Sucrose saturated fused quartz, and (B) Dry Ottawa sand (exhumed). 


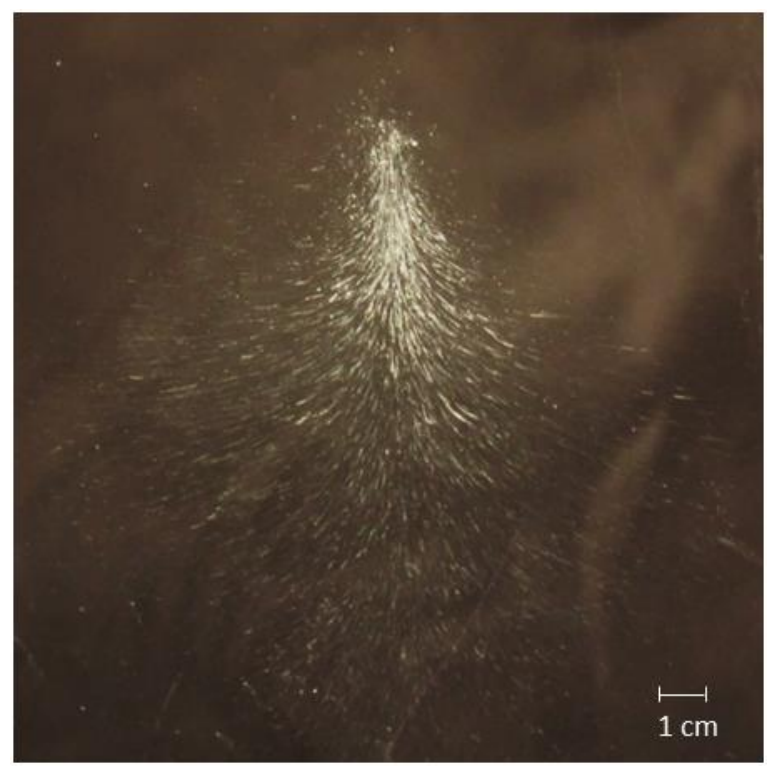

Figure 17 Gouging found on acrylic observation window after quarter space penetration event. 

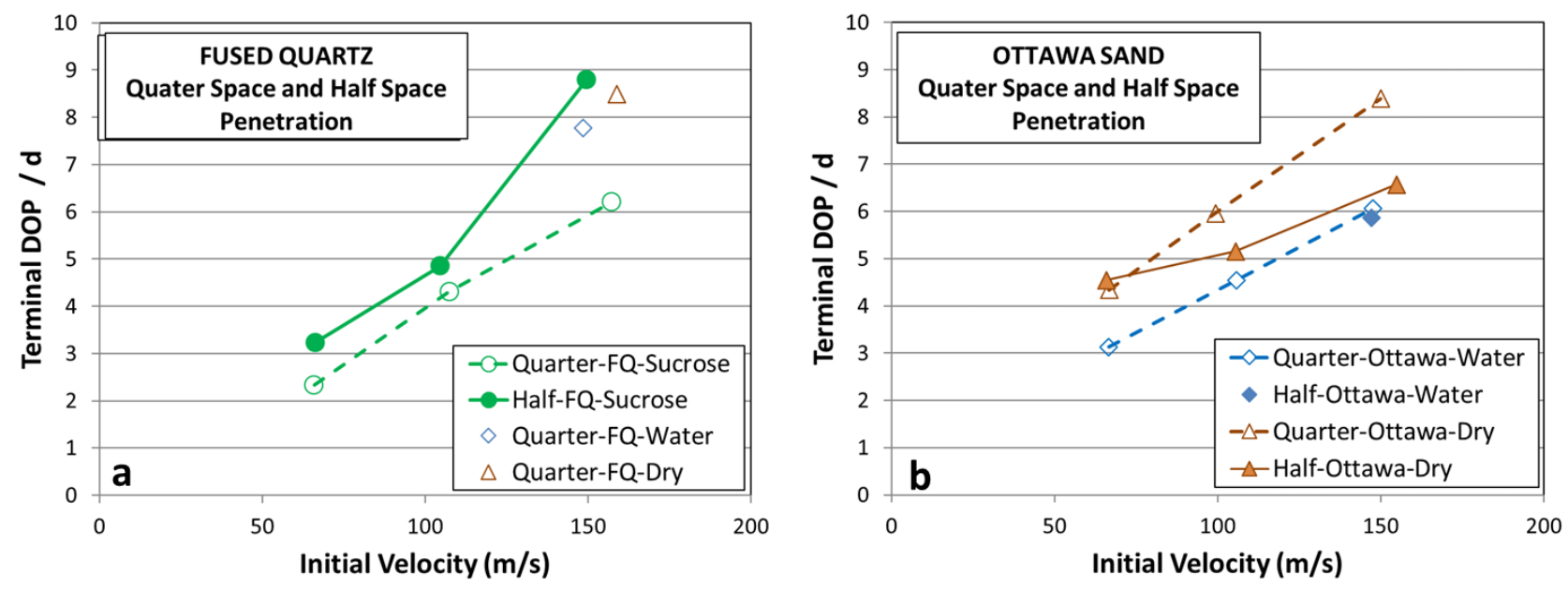

Figure 18 Initial velocity vs. terminal DOP (normalized by projectile diameter, $d$ ) for projectiles penetrating in quarter space into (A) FQ medium dense targets and (B) Ottawa Sand medium dense targets. Also, included is relevant half space penetration data from Guzman et al. 2014 


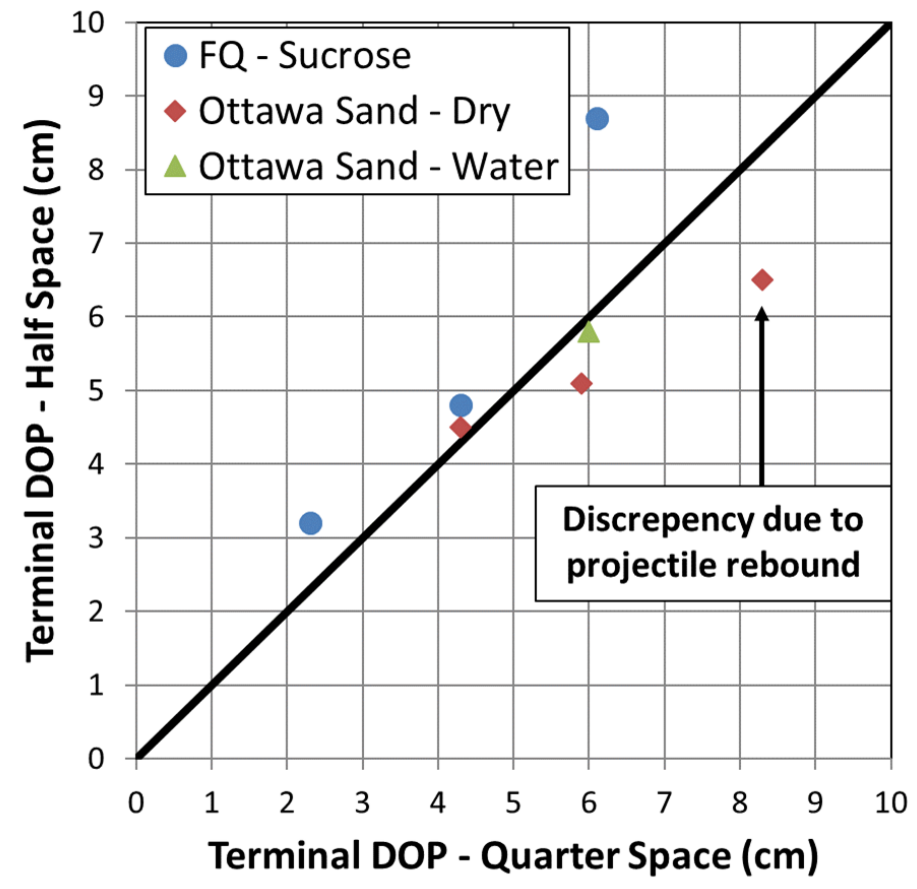

Figure 19 Comparison of terminal DOP of shots in half space vs. shots in quarter space 

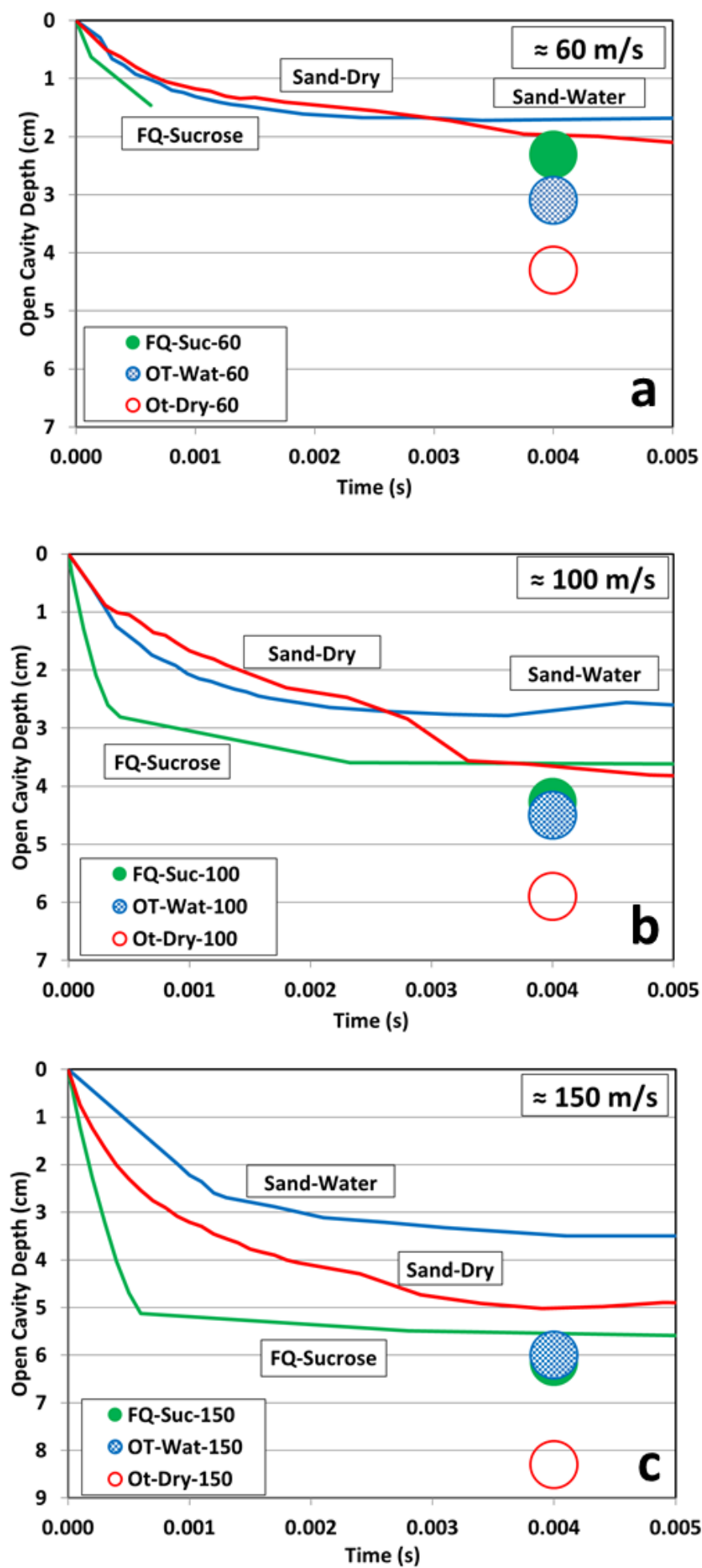

Figure 20 Depth of open cavity (as seen through the observation window) vs. time, for projectiles shot in quarter space into Ottawa sand (OT) and Fused Quartz (FQ), at approximate speeds of; (A) $60 \mathrm{~m} / \mathrm{s}$, (B) $100 \mathrm{~m} / \mathrm{s}$, and (C) $150 \mathrm{~m} / \mathrm{s}$. Vertical location of Terminal DOP is represented with big dots. 


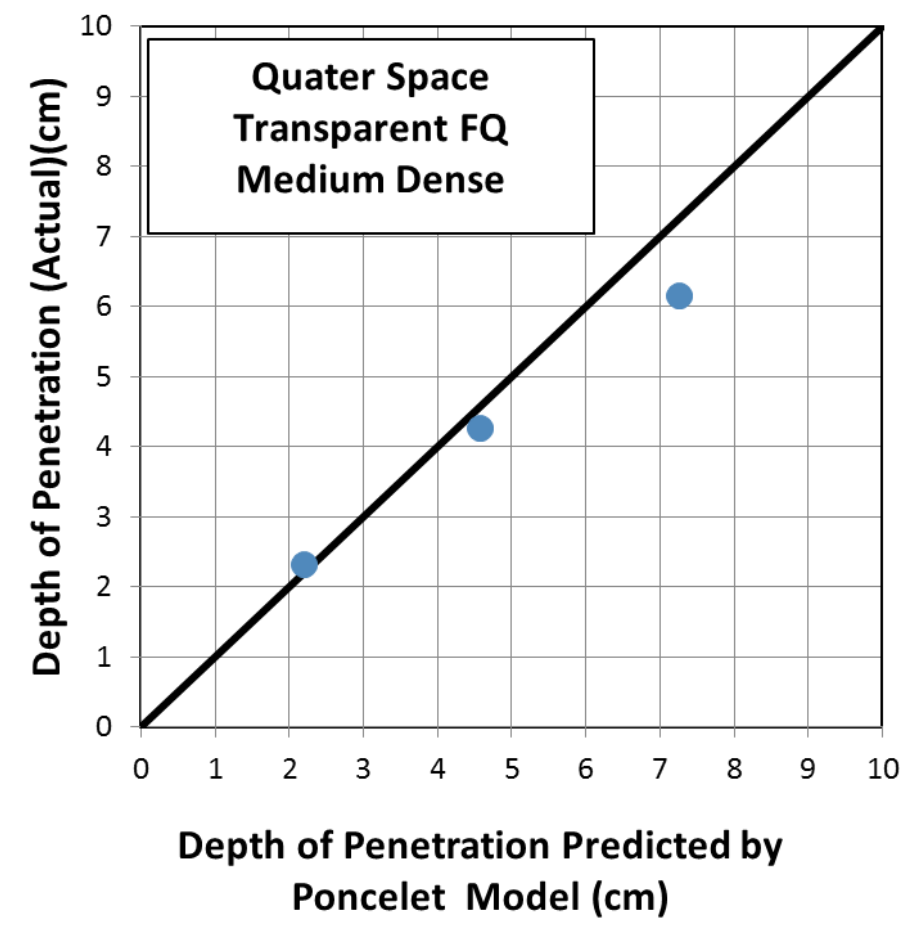

Figure 21 Comparison of actual vs. predicted (Poncelet model) terminal DOPs for sucrose saturated medium dense FQ shot in quarter space. 